\title{
BNIP3 attenuates hepatocellular carcinoma by promoting lipid droplet turnover at the lysosome.
}

\section{Damian Berardi}

The University of Chicago https://orcid.org/0000-0002-4734-5634

\section{Althea Bock-Hughes}

The University of Chicago https://orcid.org/0000-0002-5544-9740

\section{Lauren Drake}

The University of Chicago https://orcid.org/0000-0002-2022-5894

\section{Alexander Terry}

UIC https://orcid.org/0000-0003-4731-8537

\section{Grazyna Bozek}

The University of Chicago

Kay Macleod ( $\sim$ kmacleod@uchicago.edu )

The University of Chicago https://orcid.org/0000-0002-8995-4155

\section{Article}

Keywords: hepatic steatosis, lipid accumulation, tumor cell growth

Posted Date: October 11th, 2021

DOl: https://doi.org/10.21203/rs.3.rs-947988/v1

License: (c) (i) This work is licensed under a Creative Commons Attribution 4.0 International License. Read Full License 
2 BNIP3 attenuates hepatocellular carcinoma by promoting lipid droplet turnover at the lysosome.

Key words: BNIP3, mitochondria, lipogenesis

Running title: BNIP3 is a tumor suppressor in liver

Damian E. Berardi ${ }^{1}$, Althea Bock-Hughes ${ }^{1,2}$, Lauren E. Drake ${ }^{1}$, Alexander Terry ${ }^{1}$,

1 The Ben May Department for Cancer Research

The Gordon Center for Integrative Sciences, W-338

The University of Chicago

929 E $57^{\text {th }}$ Street, Chicago IL 60637

2 The Committee on Molecular Metabolism and Nutrition The University of Chicago

3 The Committee on Cancer Biology

The University of Chicago

4 Corresponding author

Tel: 773-834-8309

Fax: 773-702-4476

E-mail: kmacleod@uchicago.edu 


\section{Abstract}

32 Hepatic steatosis is a major etiological factor in hepatocellular carcinoma (HCC). Work reported here 33 identifies BNIP3 as a suppressor of HCC that mitigates against lipid accumulation. 34 Loss of BNIP3 decreased tumor latency and increased tumor burden in a mouse model of HCC. This 35 was associated with increased lipid accumulation and elevated HCC tumor cell growth. Conversely, 36 exogenous BNIP3 decreased lipid levels and reduced HCC tumor cell growth. Mutant BNIP3 ${ }^{\text {W18A }}$ that is 37 unable to promote mitophagy did not decrease HCC cell growth and was defective at reducing lipid levels. 38 Growth suppression by BNIP3 was not mediated by effects on fatty acid oxidation (FAO) or de novo 39 lipogenesis (DNL). Rather, BNIP3 suppressed HCC cell growth by promoting lipid droplet turnover at the 40 lysosome through a process we have termed "mitolipophagy" in which lipid droplets and mitochondria 41 are turned over together at the lysosome. Low BNIP3 expression in human HCC also correlated with 42 increased lipid content and worse prognosis than HCC expressing high levels of BNIP3. This work reveals 43 a role for BNIP3 and lipid droplet turnover at the lysosome in attenuating HCC. 
Introduction

The increasing incidence of hepatocellular carcinoma (HCC) in individuals with non-alcoholic fatty

48 liver disease (NAFLD) and non-alcoholic steatohepatitis (NASH) points to obesity, and to fatty liver in particular ${ }^{1-4}$, as a major etiological factor in HCC in western society ${ }^{5-10}$. Indeed, the relative risk of mortality from liver cancer was shown to be significantly elevated (1.5- to 4-fold) amongst obese patients compared to non-obese individuals, more than for any other human cancer studied ${ }^{5,11,12}$. Given the continuing increase in morbid obesity in the US population 1,10,11, efforts to understand how fatty liver promotes $\mathrm{HCC}$ are likely to be highly significant in terms of identifying biomarkers predictive of disease onset or progression, in addition to finding novel targets for therapy.

Pioneering work from the Karin lab has shown obesity driven inflammation to be a major contributing factor in the progression of NASH to HCC ${ }^{13-15}$ but defects in lipid homeostasis that cause cell death and inflammation in the liver leading to $\mathrm{HCC}$ are not fully understood. Altered mitochondrial function has been reported in human NASH and NAFLD ${ }^{16,17}$ and could contribute to altered lipid metabolism in a variety of ways, such as changes in rates of fatty acid oxidation (FAO), export of citrate to the cytosol for lipid synthesis, and production of metabolic intermediates and mitochondrial reactive oxygen species (ROS) that affect lipid species produced ${ }^{18}$. Independent of lipid metabolism, dysfunctional mitochondria also modulate production of pro-inflammatory signals, as well as the type and extent of cell death that contributes to tumor progression ${ }^{19}$. Mitochondrial function is maintained in part through the turnover of dysfunctional and/or excess mitochondria at the autolysosome by mitophagy ${ }^{20}$. BNIP3 is a mitochondrial cargo receptor that is induced in the liver by nutrient stress ${ }^{21}$ and interacts directly with processed LC3 to target mitochondria for degradation at the autolysosome thereby contributing to metabolic zonation in the liver ${ }^{22}$.

Here we examined a novel role for BNIP3 in liver tumorigenesis where we show that targeted deletion of Bnip3 in a mouse model of HCC caused reduced tumor latency and increased tumor growth rate that was associated with increased lipid content in tumors at earlier stages of disease. BNIP3 decreased HCC growth rates by increasing rates of lipid droplet (LD) turnover at the lysosome in a modified form of lipophagy that we have termed "mitolipophagy" in which LDs get turned over in

73 association with BNIP3-expressing mitochondria. Low BNIP3 expression in human HCC also correlated high lipid content and worse overall survival. 
Results

Loss of Bnip3 reduces HCC tumor latency and promotes HCC tumor growth.

To examine the role of BNIP3 in hepatocellular carcinoma (HCC), we injected 15 day old Bnip3 ${ }^{+/+}$ and bnip $3^{-/}$mice with the chemical carcinogen diethylnitrosamine (DEN) that is known to induce HCC with predicted latency in laboratory mice ${ }^{23,24}$. We harvested mice at 24,32 and 40 weeks of age to assess the effect of Bnip3 loss on both latency and growth of HCC. At 24 weeks of age, macroscopic liver lesions

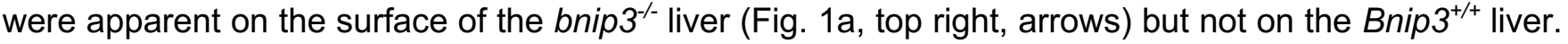
By 40 weeks of age, large tumors were obvious on both the Bnip $3^{+/+}$and bnip $3^{-/}$livers, although the

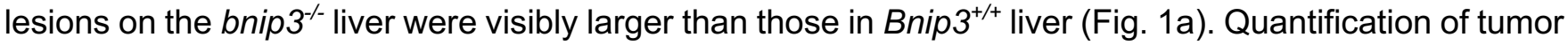
number (total per liver on serial sections through each liver) and tumor size (diameter) supported the visual assessment that tumors form earlier and grow faster in DEN-treated bnip3 ${ }^{-/-}$mice than wild-type mice. Specifically, by 24 weeks of age, there were significantly more tumors detected in the DEN-treated bnip3 $^{--}$mice than in DEN-treated wild-type control mice (Fig. 1b) although by 32 and 40 weeks of age, the difference in tumor number was no longer significant (Fig. 1b). By contrast, for those tumors that were detectable at 24 weeks, there was no significant difference in tumor size between wild-type and bnip $3^{-/-}$ mice initially but by 32 and 40 weeks, the bnip3 $3^{-/-}$tumors were significantly larger than those forming in wild-type mice (Fig. 1c). In summary, loss of Bnip3 reduces tumor latency and increases tumor growth rate of DEN-induced HCC in mice.

Interestingly, when we examined BNIP3 expression by immunohistochemistry in tumors forming in wild-type mice, we noted that BNIP3 expression is elevated in HCC tumors (T) at 24 weeks compared to adjacent normal (N) liver (Fig. 1d). By contrast, BNIP3 levels were lower in HCC tumors (T) compared to adjacent normal liver (N) at 32 weeks and 40 weeks (Fig. 1d). The upregulation of BNIP3 in HCC tumors at 24 weeks compared to adjacent normal liver, is likely mediated by elevated nuclear Hif $1 \alpha$ expression which is also elevated in HCC tumors at 24 weeks (Supp. Fig. 1). qPCR showed that Bnip3 mRNA isolated from primary tumor and adjacent normal tissue was down-regulated in tumors at 32 weeks and 40 weeks of age (Fig. 1e) suggesting that loss of BNIP3 expression in wild-type HCC at later

101 timepoints is likely mediated by gene silencing since Bnip3 mRNA levels are decreased (Fig. 1e) despite persistent expression of nuclear Hif1 $\alpha$ (Supp. Fig. 1). Consistent with RNA expression analysis, most HCC tumors at 32 weeks and all tumors at 40 weeks showed decreased BNIP3 protein levels, compared to levels in adjacent normal liver (Fig. 1f). These results show that while BNIP3 is expressed at higher 
109 In addition to loss of BNIP3 expression in wild-type tumors at 32 to 40 weeks of age, there was also a 110 change in the appearance of HCC tumors forming in wild-type mice. At 24 weeks of age, wild-type HCCs 111 contain small, tightly packed tumor cells, whereas by 32 and 40 weeks of age, HCC tumors contained 112 larger tumor cells with more "bubbles" suggesting increased lipid accumulation (Fig. 1d). To assess this, 113 we stained liver sections from wild-type and bnip ${ }^{-/-}$DEN treated mice with Oil red O (ORO) to determine 114 lipid content in tumors at 24, 32 and 40 weeks (Fig. 2a-c). Consistent with the pattern detected by H\&E 115 staining (Fig. 1d; Fig. 2a, top left), we observed that wild-type tumors at 24 weeks contained less lipid 116 compared to surrounding normal tissue (Fig. 2a, middle panel). By contrast, HCC tumors forming in bnip3 117 / livers contained considerably more lipid at 24 weeks than did tumors in wild-type mice (Fig. 2a) which 118 was apparent by both H\&E staining and by ORO staining. However, tumors growing in 40 week old wild119 type mouse liver that had lost Bnip3 expression (Fig. 1d-f), exhibited higher lipid content than surrounding normal liver and as high as that detected in bnip3 $3^{-/}$tumors (Fig. 2b). Quantification of ORO staining confirmed these observations (Fig. 2c). At 24 weeks, there was increased lipid content in bnip $3^{-/-}$tumors compared to wild-type, but these differences in lipid content diminished over time at 32 and 40 weeks, as wild-type tumors lost Bnip3 expression and simultaneously accumulated more lipid (Fig. 2c). Increased lipid content in bnip $3^{-/}$HCC was associated with increased transcript levels of genes involved in fatty acid synthesis (Fig. 2d), including fatty acid synthase (Fasn), acetyl CoA carboxylase 1 (Acaca), ATP citrate lyase (Acly) and Stearoyl CoA desaturase-1 (Scd1). Immunohistochemistry for ACACA and FASN indicated that HCC tumors at 24 weeks in both $\mathrm{Bnip}^{+/+}$and bnip $3^{-/-}$mice express higher levels of these enzymes than surrounding normal tissue (Fig. 2e-f). In summary, our findings show that loss of Bnip3 either by genetic deletion in bnip $3^{-/}$mice, or via silencing at late stages of tumorigenesis in wild-type mice is associated with increased lipid accumulation in HCC.

\section{BNIP3 reverses lipid accumulation in HCC cells and inhibits HCC cell growth.}

To gain mechanistic insight to how BNIP3 was modulating lipid content in HCC tumors, we established primary cell lines from different bnip $3^{-/}$tumors and then generated stable lines using lentiviral vectors expressing either empty vector (EV), HA-tagged wild-type BNIP3 (HA-BNIP3) or HA-tagged BNIP3 mutated at W18 to inhibit LC3 interaction and block mitophagy (HA-BNIP3 $\left.{ }^{\mathrm{W} 18 \mathrm{~A}}\right)^{22}$. We validated expression of EV, HA-BNIP3 or HA-BNIP3 ${ }^{\mathrm{W} 18 \mathrm{~A}}$ in bnip3 $3^{-/}$lines (Fig. 3a) and confirmed expression of

138 alpha-fetoprotein (AFP), an oncofetal protein expressed during liver development and re-expressed in 139 HCC tumors (Fig. 3a). We confirmed that expression of HA-BNIP3, but not EV or HA-BNIP3 ${ }^{\text {W18A }}$, 140 promoted mitophagy by staining for overlap between processed LC3B (an autophagosome marker) and 141 TOMM20 (a mitochondrial marker) in the presence of bafilomycin $\mathrm{A}_{1}$ (Fig. 3b). Exogenous HA-BNIP3 142 expression decreased staining for TOMM20 and induced significant overlap in LC3B-TOMM20 staining, 
143 whereas EV and HA-BNIP3 ${ }^{\text {W18A }}$ did not (Fig. 3b). Decreased mitochondrial staining was visible in HA144 BNIP3 expressing HCC cells in the presence (Fig. 3b) or absence of bafilomycin $\mathrm{A}_{1}$ (Supp. Fig. 2a), but 145 the overlap between LC3 and TOMM20 could only be detected in the presence of bafilomycin $A_{1}$ (Fig. $1463 \mathrm{~b}$ ) indicating that there was increased mitophagic flux when HA-BNIP3 was expressed but not with EV 147 or HA-BNIP3 ${ }^{\text {W18A }}$ expression. We also noted that HA-BNIP3 induced mitochondrial fragmentation, that 148 was most striking when cells were bafilomycin $A_{1}$ treated (Fig. 3b), but not in the absence of bafilomycin 149 A1 (Supp. Fig. 2a). Together these results indicate that BNIP3 promotes mitochondrial fragmentation and 150 preferential mitophagy of these fragmented mitochondria at the autolysosome, which is dependent on its 151 interaction with LC3, since this was not observed in either EV or HA-BNIP3 ${ }^{\text {W18A }}$ expressing HCC cells.

152 Mitochondrial mass was also indirectly assessed by qPCR for mitochondrial genome copy 153 number (amplifying for $N d 1$ and Cytb) relative to nuclear DNA (amplifying beta-hemoglobin/Hbb) and 154 shown to be decreased in HCC cells expressing HA-BNIP3 but not EV or HA-BNIP3 ${ }^{\text {W18A }}$ (Fig. 3c). Also, 155 consistent with decreased mitochondrial mass, we observed decreased oxygen consumption in HCC cells expressing HA-BNIP3 but not EV or HA-BNIP3 ${ }^{\text {W18A }}$ (Fig. 3d). Interestingly, we observed that exogenous HA-BNIP3 decreased extracellular acidification of growth media following a glycolysis stress test performed using the Seahorse analyzer (Fig. 3e) and suppressed glucose uptake by HCC cells in culture (Fig. 3f). Consistent with these findings in vitro and with increased growth rate of HCC lacking 160 Bnip3, there was increased glucose uptake into HCCs in bnip3 $3^{-/}$liver compared to Bnip3 ${ }^{+/+}$liver as measured by FDG-PET analysis of live mice at 32 weeks of age (Supp. Fig. 3). Overall, these results show that exogenous HA-BNIP3, but not EV or HA-BNIP3 ${ }^{\text {W18A }}$, promotes mitophagy and reduces 163 mitochondrial mass in HCC cells. In addition, BNIP3 reduces reliance on glucose to fuel either OXPHOS 164 or glycolysis.

165 Given the steatotic phenotype in vivo in HCC tumors growing in the absence of BNIP3, we examined lipid content of HCC cells in culture expressing EV, HA-BNIP3 or HA-BNIP3 ${ }^{\text {W18A }}$ by staining cells with the lipophilic dye, BODIPY 493/503. We observed that expressing exogenous HA-BNIP3 decreased the number of BODIPY positive lipid droplets in HCC cells compared to EV control expressing HCC cells (Fig. 4a-4c). Intriguingly, even though HA-BNIP3 ${ }^{\mathrm{W} 18 \mathrm{~A}}$ was unable to promote mitophagy, we observed that this mutant form of BNIP3 retained ability to decrease lipid droplet number compared to 171 EV (Fig. 4a-b). However, when we challenged these cells with oleic acid to further increase cellular lipid 172 content, HA-BNIP3 was significantly more effective at decreasing lipid droplet number than either EV or 173 HA-BNIP3 $^{\text {W18A }}$ (Fig. 4a, 4c). Nevertheless, HA-BNIP3 ${ }^{\text {W18A }}$ retained partial ability to decrease lipid droplet 174 number suggesting that while mitophagy is involved in how BNIP3 limits lipid accumulation, a second 175 mitophagy-independent role is also at play. We noted that while HA-BNIP3 ${ }^{\mathrm{W} 18 \mathrm{~A}}$ is defective for mitophagy, 176 it retains the ability to induce mitochondrial fragmentation raising the possibility that mitochondrial 
177 fragmentation contributes to how BNIP3 prevents lipid accumulation. Analysis of BODIPY staining of HCC cells by Imagestream flow cytometric analysis confirmed results obtained by immunofluorescence microscopy in showing decreased lipid droplet numbers when HA-BNIP3 was expressed but not EV, and less so with HA-BNIP3 ${ }^{\text {W18A }}$ (Fig. 4d, 4e). Imagestream analysis also showed HCC cells expressing HABNIP3 to be smaller in size that HCC cells expressing EV or HA-BNIP3 ${ }^{\text {W18A }}$ (Fig. 4d, 4f). Previous work has linked lower mitochondrial mass and function to decreased overall cell size ${ }^{25,26}$.

Exogenous expression of HA-BNIP3 in bnip ${ }^{-/} \mathrm{HCC}$ cells also decreased expression of lipogenic genes (Fasn, Acaca, Acly) (Fig. 4g). Most interestingly, exogenous HA-BNIP3 markedly slowed down the growth of bnip $3^{-/-}$HCC cells whereas EV and HA-BNIP3 ${ }^{\text {W18A }}$ did not (Fig. 4h). In summary, BNIP3 induced mitochondrial fragmentation and mitophagy, decreased lipid accumulation and decreased growth of HCC cells in culture.

\section{BNIP3 promotes fatty acid oxidation but this does not explain how BNIP3 decreases lipid levels} or inhibits tumor cell growth.

Lipid accumulation in cells can arise for various reasons, including decreased fatty acid oxidation (FAO) ${ }^{27}$ and since FAO takes place in the mitochondrial matrix, we postulated that BNIP3 may be promoting lipid turnover by increasing FAO. To examine this, we measured oxygen consumption in HCC cells expressing EV, HA-BNIP3 or HA-BNIP3 ${ }^{\mathrm{W} 18 \mathrm{~A}}$ using palmitate as a substrate for oxidation (Fig. 5a-e). Given that exogenous HA-BNIP3 decreased mitochondrial mass (Fig. 3b, 3c) and lowered oxidation of glucose in bnip3 ${ }^{-/}$cells (Fig. 3d), we were surprised to observe that HA-BNIP3 markedly increased FAO of palmitate in bnip3 $3^{--}$HCC cells compared to EV or HA-BNIP3 ${ }^{\text {W18A }}$ (Fig. 5a). HA-BNIP3 expressing HCC cells were also more sensitive to etomoxir (ETO) which suppresses carnitine palmitoyl transferase-1 (CPT1) and blocks fatty acid uptake into the mitochondria to suppress FAO (Fig. 5b, Fig. 5d). Indeed, ETO collapsed oxygen consumption by HA-BNIP3 expressing HCC cells using palmitate as substrate, down to levels seen in HCC cells expressing EV or HA-BNIP3 ${ }^{\text {W18A }}$ (Fig. 5b, 5d). By contrast ETO had no effect on HCC cells expressing EV or HA-BNIP3 ${ }^{\mathrm{W} 18 \mathrm{~A}}$ (Fig. 5c, 5e). These results suggest that HA-BNIP3 is promoting $\mathrm{FAO}$ and thus we were interested to determine if this explained how HA-BNIP3 was able to promote lipid turnover in HCC cells.

Using similar approaches to those described above, we examined whether inhibiting FAO with ETO inhibited the ability of HA-BNIP3 to decrease numbers of BODIPY-positive lipid droplets in HCC cells. Surprisingly, even in the presence of ETO that clearly disrupted FAO, HA-BNIP3 retained the ability to decrease lipid droplet content in HCC cells, even when FAO was inhibited (Fig. $5 \mathrm{f}, 5 \mathrm{~g}$ ). This ability was also still evident in HCC cells fed oleic acid in the presence of ETO (Fig. 5f, 5h), suggesting that while HA-BNIP3 does indeed promote FAO, HA-BNIP3 has a second activity that regulates lipid droplet content 
211 in HCC cells. Significantly, HA-BNIP3 ${ }^{\text {W18A }}$ was also able to decrease lipid droplet number in the presence

212 of Etomoxir (Fig. 5f, Fig. 5g), even though this form of BNIP3 was unable to promote FAO of palmitate

213 (Fig. 5b, Fig. 5e). Again, this effect of BNIP3 ${ }^{\mathrm{W} 18 \mathrm{~A}}$ was overcome when cells were fed oleic acid to increase

214 lipid droplet content such that it was clearly less effective than wild-type BNIP3 at promoting lipid droplet 215 clearance (Fig. 5f, Fig. 5g).

216 Expression of certain genes involved in FAO were modestly increased in HCC cells expressing 217 HA-BNIP3 compared to either EV expressing or HA-BNIP3 ${ }^{\text {W18A }}$ expressing cells (Fig. 5i) but again, since 218 effects on FAO do not appear to explain the ability of BNIP3 to decrease lipid levels in cells, it is unclear 219 what the significance of the increased expression of CPT2, ACADM and ACADL are for cell growth. Indeed, treatment of HA-BNIP3 expressing HCC cells with ETO had no effect on cell growth (Fig. 5j). These results show that while BNIP3 inhibits FAO in bnip3 $3^{-/-} \mathrm{HCC}$ cells, this does not explain how BNIP3 promotes decreased lipid content or how BNIP3 suppresses HCC cell growth.

\section{BNIP3 does not decrease lipid levels or HCC cell growth via effects on lipogenesis.}

To assess whether BNIP3 was decreasing lipid content in HCC cells by inhibiting de novo lipogenesis, we examined the effect of treating cells with an active site inhibitor of FASN called TVB-3664 that is active on mouse cells ${ }^{28}$. Treatment of cells for 24 hours with TVB-3664 markedly increased expression of fatty acid synthesis genes (Fasn, Acaca, Acly) irrespective of genotype (Fig. 6a), as expected due to transcriptional feedback effects when lipid synthesis is repressed ${ }^{29}$. Inhibiting FASN is expected to increase cellular levels of malonyl CoA that gets depleted as a substrate for FASN to make palmitate. Interestingly, only cells expressing HA-BNIP3, not EV or HA-BNIP3 ${ }^{\mathrm{W} 18 \mathrm{~A}}$, showed increased malonyl CoA when treated with the TVB-3664 FASN inhibitor (Fig. 6b). This suggests that FASN was more active in HA-BNIP3 expressing HCC cells than cells expressing either EV or HA-BNIP3 ${ }^{\mathrm{W} 18 \mathrm{~A}}$, consistent with there being lower lipid levels in cells expressing HA-BNIP3. In addition to acting as a substrate for FASN, malonyl CoA also inhibits FAO by preventing carnitine uptake by CPT ${ }^{30}$. Similar to the effects of ETO on FAO, and consistent with increased malonyl CoA produced by FASN inhibition in HA-BNIP3 expressing cells, we observed that TVB-3664 inhibited FAO of palmitate in HA-BNIP3 expressing HCC cells (Fig. 6d) compared to EV (Fig. 6c) or HA-BNIP3 ${ }^{\text {W18A }}$ expressing cells (Fig. 6e). Thus, TVB-3664 inhibits both lipid synthesis and FAO in HA-BNIP3 expressing bnip3 ${ }^{-/}$HCC cells.

When we examined the effect of TVB-3664 on lipid content of HCC cells, we observed that it eliminated lipid content almost completely independent of BNIP3 since it decreased lipid content in cells expressing EV, HA-BNIP3 or HA-BNIP3 ${ }^{\mathrm{W} 18 \mathrm{~A}}$ to undetectable levels (Fig. 6f, Fig. 6h). However, by

243 increasing the amount of lipid in cells by feeding them with oleic acid, we observed that HA-BNIP3, but 244 not EV or HA-BNIP3 ${ }^{\mathrm{W} 18 \mathrm{~A}}$, remained able to decrease lipid content even when FASN (and FAO) was 
245 inhibited (Fig. 6g, Fig. 6h), arguing that the ability of BNIP3 to decrease lipid content in HCC cells was 246 independent of effects on DNL and/or FAO. Furthermore, the repressive effect of FASN inhibition on 247 growth of HA-BNIP3 expressing cells was synergistic with the effect of exogenous HA-BNIP3 (Fig. 6i) 248 suggesting that BNIP3 is suppressing tumor cell growth in a manner independent of lipid synthesis. In 249 addition, TVB-3664 inhibition of FASN was also able to inhibit growth of EV and HA-BNIP3 ${ }^{\text {W18A }}$ expressing HCC cells (Fig. 6i), consistent with the growth suppressive effects of TVB-3664 being BNIP3independent. In summary, while TVB-3664 inhibits HCC cell growth, this is independent of BNIP3 and BNIP3 (but not EV or BNIP3 ${ }^{\mathrm{W} 18 \mathrm{~A}}$ ) suppresses HCC cell growth independently of TVB-3664 and FASN activity. Thus far, we have excluded the effects of BNIP3 on FAO or DNL as explaining how BNIP3 decreases lipid levels and attenuates HCC tumor cell growth.

\section{BNIP3 limits lipid content and HCC cell growth by promoting lipid droplet turnover.}

Another mechanism by which cells regulate lipid content is via turnover of lipid droplets (LDs) that involves both lipolysis and lipophagy. LDs are a means of storing triacylglycerides, stearyl esters and retinyl esters within a phospholipid membrane and LDs play important roles in the regulation of fatty acid trafficking within the cell ${ }^{31}$. LD turnover relies on cytosolic lipases, such as adipose triglyceride lipase (ATGL) while the engulfment of LDs by autophagosomes in a process known as lipophagy, relies on acid lipases at the lysosome ${ }^{32-34}$. LDs are known to associate with mitochondria ${ }^{35-37}$ and given the role of BNIP3 in mitophagy, we examined a potential role for BNIP3 in promoting LD turnover via a mechanism that integrates mitophagy with lipophagy. When we inhibited lysosomal lipases with Lalistat2 (LALi) ${ }^{33}$, we observed increased numbers of LDs accumulating in HCC cells when HA-BNIP3 was expressed but less with EV or HA-BNIP3 ${ }^{\text {W18A }}$ (Fig. 7a, 7b) suggesting that BNIP3 relied on lysosomal lipases to elicit at least part of its effect in decreasing LD numbers. ImageStream analysis confirmed that LALi only increased BODIPY LD number significantly when HA-BNIP3 was expressed and less so when HABNIP3 ${ }^{\mathrm{W} 18 \mathrm{~A}}$ or EV was expressed (Supp. Fig. 4a, 4b). Interestingly, LALi appeared to increase LD numbers but did not increase overall cell size of HCC cells expressing HA-BNIP3, (Supp. Fig. 4a, 4c) indicating that reduced HCC cell size with HA-BNIP3 was not likely due to effects of BNIP3 on LD numbers. In summary, these results suggested to us that BNIP3 was decreasing lipid content in HCC cells by promoting LD turnover at the lysosome.

To examine this more carefully, we co-stained cells for BODIPY and lysotracker to examine overlap of LDs with the lysosome. Experiments were performed in the presence of oleic acid and LALi to allow such structures to accumulate. While a few overlapping LD/lysosomes were detected in EV and HA-BNIP3 ${ }^{\text {W18A }}$ expressing HCC cells (Fig. 7c), there was a marked increase in overlap (white) between 278 BODIPY (green) and Lysotracker (magenta) in HCC cells expressing HA-BNIP3 (Fig. 7c). Notably, 
smaller green LDs were seen predominantly inside magenta-colored lysosomes while larger LDs did not associate with lysosomes (Fig. 7c). Analysis of LDs, mitochondria and lysosomes in the different HCC lines by transmission electron microscopy (TEM), revealed plentiful LDs in both EV and HA-BNIP3 ${ }^{\text {W18A }}$ expressing cells (Fig. 7d), and fewer LDs in the HA-BNIP3 expressing HCC cells, consistent with BODIPY imaging of cells showing HA-BNIP3 decreasing LD number. There were also more mitochondria in EV and HA-BNIP3 ${ }^{\text {W18A }}$ expressing HCC cells (Fig. 7d), supporting conclusions from Fig. 3 that HA-BNIP3, but not EV or HA-BNIP3 ${ }^{\mathrm{W} 18 \mathrm{~A}}$, promotes mitophagy and lowers mitochondrial mass. Lysosomes were also more evident in HA-BNIP3 expressing cells (Fig. 7d) and contained LD-like structures inside lysosomes (Fig. 7e, middle) as also seen by fluorescence microscopy (Fig. 7e, left) as well as LDs associated with mitochondria (Fig. 7e, right). Taken together, these data suggest that BNIP3 is promoting LD turnover at the lysosome in conjunction with turnover of mitochondria.

Treatment of cells with LALi significantly decreased the growth of HCC cells expressing HA-BNIP3 but had no effect on the growth of cells expressing EV or HA-BNIP3 ${ }^{\text {W18A }}$ (Fig. 7f), suggesting that BNIP3 lipases are fueling FAO in HCC cells (Fig. 7g). However, this effect was independent of BNIP3 consistent with data described above (Fig. 5) indicating that altered FAO does not explain how BNIP3-dependent LD turnover suppresses cell growth. At this time, it is not clear to us how LDs promote HCC cell growth but LDs could act as reservoirs of phospholipids and other lipids used by growing cancer cells for membrane expansion and other pro-growth functions. In summary, these data indicate that BNIP3 attenuates HCC cell growth by promoting LD turnover by a process we refer to as "mitolipophagy".

\section{Low BNIP3 expression in human HCC correlates with worse overall survival.}

We examined publicly available RNA-Seq data comparing transcript expression in human HCC

303 to healthy human liver tissue ${ }^{38}$, that showed expression of genes involved in fatty acid metabolism, 304 cholesterol metabolism and adipogenesis to be up-regulated in HCC compared to healthy liver (Supp. 305 Fig. 5a-c), in line with previously published data ${ }^{39-41}$. When we examined BNIP3 expression in these 306 data-sets, we observed that while lipid synthesis genes like FASN and ACLY were increased in HCC 307 compared to healthy tissue, BNIP3 was significantly decreased (Fig. 8a, 8b). Interestingly, PPARGC1A 308 that promotes mitochondrial biogenesis was also increased in HCC compared to healthy liver while 309 ACADM that promotes FAO was decreased (Fig. 8a). Linear regression analysis showed a highly 310 significant inverse correlation between levels of FASN, ACLY and PPARGC1A and BNIP3 (Supp. Fig. $3115 \mathrm{~d}-\mathrm{f}$ ) and a direct correlation between ACADM and BNIP3 (Supp. Fig. 5g). These data indicate that in 312 HCC where lipogenic programs are increased, BNIP3 is significantly decreased. 
We further explored TCGA data looking at how expression levels of BNIP3 affects overall survival

314 of patients with HCC and showed a correlation between levels of BNIP3, acetyl CoA carboxylase-1

315 (ACACA) and patient survival. The ACACA gene encodes an enzyme that catalyzes conversion of acetyl

316 CoA to malonyl CoA to promote lipid synthesis, and is frequently up-regulated in human cancers,

317 including HCC ${ }^{42,43}$. Interestingly, we found that high BNIP3 expression levels in combination with high

318 ACACA expression portended a highly significant increase in overall survival rates (Fig. 8c) in contrast

319 to the combination of high ACACA expression with low BNIP3 expression that had the worst prognosis

320 for overall survival (Fig. 8e). Moreover, when we examined the histology of liver sections from these HCC

321 patients, we observed that high BNIP3 mitigated against lipid accumulation linked to high ACACA

322 expression (Fig. 8d) in contrast to low BNIP3 where high levels of lipid were associated with high ACACA

323 expression (Fig. 8f). These results indicate that high BNIP3 expression is associated with decreased lipid

324 accumulation, less aggressive HCC and increased patient survival when linked to high ACACA

325 expression while conversely low BNIP3 predicts increased lipid accumulation and worse patient outcome. 


\section{Discussion}

Our work identifies a novel role for BNIP3 in limiting HCC by promoting lipid droplet turnover at the lysosome (Fig. 9). This conclusion was reached after first interrogating the role of BNIP3 in rates of fatty acid oxidation (FAO) and also in de novo lipogenesis (DNL). FAO in particular seemed likely to be influenced by BNIP3 since it takes place in the mitochondrial matrix. FAO has generally been reported

332 to be required for cancer growth, including in MYC-driven triple negative breast cancer ${ }^{44}$, KRAS-driven 333 lung cancer ${ }^{45}$ and other cancers ${ }^{46}$. FAO protects tumor cells from cell death induced by loss of 334 attachment (anoikis) ${ }^{47}$ and/or under conditions of nutrient deprivation ${ }^{48}$. In these conditions, FAO335 dependent generation of ATP for energy, $\mathrm{NADH}$ to feed the electron transport chain, acetyl CoA to prime the TCA cycle and NADPH (via TCA-derived citrate) to mitigate against ROS levels, have all been cited as explaining the pro-tumorigenic function of FAO ${ }^{49}$. To our surprise, BNIP3 very potently promoted FAO in $\mathrm{HCC}$ cells using palmitate as a substrate (Fig. $5 \mathrm{~d}$ ) whereas mitophagy-defective BNIP3 ${ }^{\mathrm{W} 18 \mathrm{~A}}$ was unable to promote FAO (Fig. 5e) indicating that the strong effect of BNIP3 on FAO was mitophagy-dependent. Mitochondrial fission is known to precede mitophagy ${ }^{50,51}$ and indeed, BNIP3 induces both mitochondrial fragmentation and mitophagy ${ }^{52}$. Thus, one possible explanation for how BNIP3 promotes FAO is by promoting the turnover of smaller more fragmented mitochondria, thereby increasing the cellular proportion of fused mitochondria. Fused mitochondria are more efficient at FAO ${ }^{53}$, possibly due to more efficient exchange of reducing agents and metabolic intermediates within the mitochondrial matrix ${ }^{54}$. 345 Nevertheless, this effect of BNIP3 in promoting FAO is unlikely to explain HCC growth suppression since inhibition of FAO with Etomoxir failed to block the growth suppressive properties of BNIP3 (Fig. 5j).

Increased rates of de novo lipogenesis (DNL) have also been linked to increased tumorigenesis, including HCC and other cancers ${ }^{43,55}$, and indeed SNPs associated with the FASN, ACACA and ACLY genes predict disease outcome including recurrence of human $\mathrm{HCC}{ }^{56}$. Increased lipid synthesis may promote tumor cell growth by fueling triglyceride formation for cell membranes and signaling, amongst other functions ${ }^{27}$. Interestingly, breast cancer cells are particularly dependent on fatty acid synthesis after metastasizing to the brain which has limited fatty acid availability compared to either the primary site or other metastatic sites ${ }^{57}$. These studies have driven efforts to target FASN and ACACA enzymes for therapeutic benefit ${ }^{43,55,58-60}$. However, our work shows that the ability of BNIP3 to promote lipid clearance from HCC tumor cells, was not dependent on rates of lipogenesis since BNIP3 could decrease lipid levels robustly even in the presence of an effective FASN inhibitor (Fig. 6h).

Having excluded effects of BNIP3 on FAO and DNL as explaining how BNIP3 decreased both lipid

358 droplet (LD) number and HCC cell growth, we considered the possibility that BNIP3 was promoting 359 lipolysis of neutral lipids contained in LDs. Inhibiting lysosomal acid lipases with Lalistat2 (LALi) 360 significantly attenuated the inhibitory effect of BNIP3 on LD number (Fig. 7a) suggesting that BNIP3 is 
361 somehow promoting lipophagy in which lipid droplets are turned over at the lysosome ${ }^{32}$. This became 362 more interesting when, unlike FAO and DNL inhibitors, we also observed a marked decrease in the ability 363 of BNIP3 to limit HCC tumor cell growth following treatment with LALi (Fig. 7f). Using LALi to inhibit 364 turnover of LDs that had been taken up by lysosomes, we observed that BNIP3 promoted increased LD 365 uptake by lysosomes compared to either EV or BNIP3 ${ }^{\mathrm{W} 18 \mathrm{~A}}$, but also that it was the smallest LDs that were 366 taken up by the lysosome (Fig. 7c). Larger LDs appeared to be resistant to turnover at the lysosome. As 367 discussed above, mitophagy is preceded by mitochondrial fragmentation and there is preferential 368 turnover of smaller mitochondria causing larger more fused mitochondria to predominate ${ }^{50,51 .}$ 369 Mitochondria and LDs associate with each other through various mechanisms ${ }^{35,36}$, and LD size is partly 370 determined by surface area of contact with peri-droplet mitochondria ${ }^{61}$. LD size increases in proportion 371 to the size of the mitochondria with which it associated ${ }^{61}$. Conversely, smaller lipid droplets associate 372 with smaller mitochondria that in turn are more susceptible to turnover at the autolysosome.

Thus, to reconcile our collective findings, we suggest that BNIP3 promotes LD turnover through 374 "mitoplipophagy" in which small LDs get turned over with associated smaller, fragmented mitochondria 375 (Fig. 9). Selective forms of autophagy imply that only the selected cargo gets turned over and certainly 376 upregulation of specific cargo receptors like BNIP3 promote increased mitophagy preferentially over other selective forms of autophagy. However, analysis of electron micrographs clearly indicates that while 378 mitochondria make up the bulk of the cargo during mitophagy, other organelles, such as associated ER 379 and ribosomes also get turned over by association with targeted mitochondria ${ }^{62}$. Indeed, our TEM 380 revealed interactions between fragmented mitochondria and lipid droplets inside membrane bound vesicles (Fig. 7e), consistent with our suggestion that BNIP3 limits lipid accumulation by promoting LD

382 turnover with associated mitochondria. This BNIP3-dependent tumor suppressive mechanism may be 383 unique to liver where accumulating lipid promotes both NAFLD and NASH, and progression to HCC, and 384 ongoing studies are examining the extent to which BNIP3 modulates lipid content in other tissues and 385 tumor types, and whether this contributes to the growth suppressive effect of BNIP3. 


\section{Methods}

391 Mice

392 All mice (wild-type and bnip $3^{-/-}$mice) were maintained on a pure C57BI/6J genetic background. Mice were

393 maintained in an environmentally controlled specific pathogen free barrier facility and provided ad libitum

394 with water and chow. Tumors were induced in mice by intra-peritoneal injection of 15 day old male mice

395 with $25 \mathrm{mg} / \mathrm{kg}$ diethylnitrosamine (DEN).

\section{Cells}

398 Primary hepatocellular carcinoma cell lines were established from bnip3 $3^{-/-}$mice using a standard twostep liver perfusion technique, as described previously by our lab ${ }^{22}$. Macroscopic tumors evident in the excised and collagenase perfused liver were dissected away from surrounding non-tumor liver parenchyma in a culture dish containing isolation media (DMEM/4.5 g/l glucose, $1 \mathrm{mM}$ lactate, $2 \mathrm{mM} \mathrm{L-}$ glutamine, $15 \mathrm{mM}$ Hepes, $100 \mathrm{nM}$ dexamethasone, $10 \%$ defined fetal bovine serum (Hyclone), $100 \mathrm{U} / \mathrm{ml}$ penicillin $/ 0.1 \mathrm{mg} / \mathrm{ml}$ streptomycin). Tumors were disaggregated by pipetting, filtered through a $75 \mu \mathrm{m}$ filter and washed three times in isolation media. The HCC cell pellet was resuspended in defined HCC growth media (DMEM/F12, 10\% defined fetal bovine serum (Hyclone), $100 \mathrm{nM}$ dexamethasone, $20 \mu \mathrm{g} / \mathrm{l}$ epidermal growth factor, 1x Insulin-Transferrin-Selenium (ITS, Gibco, Cat\# 41400045), 100 U/ml penicillin $/ 0.1 \mathrm{mg} / \mathrm{ml}$ streptomycin). HCC cell lines were expanded and infected overnight in the presence of $8 \mu \mathrm{g} / \mathrm{ml}$ polybrene with lentivirus ( $\mathrm{pLVX}$ ) expressing either empty vector sequences (EV), HA-BNIP3 or HA-BNIP3 ${ }^{\mathrm{W} 18 \mathrm{~A}}$, and selection for expressing lines was performed in $200 \mu \mathrm{g} / \mathrm{ml}$ hygromycin. Cell lines were validated for exogenous BNIP3 expression by western blot with $\alpha-\mathrm{HA}, \alpha-A F P$ and by immunofluorescence for HA (see below). Experiments were conducted in HCC defined media (no

412 hygromycin) in the presence or absence of $100 \mu \mathrm{M}$ BSA-conjugated oleic acid (Sigma cat\# O3008). Drug 413 treatments included $10 \mu \mathrm{M}$ Etomoxir (Sigma cat\# 509455) for 24 hours, 12 nM TVB-3664 (3V414 Biosciences) for 24 hours, $20 \mu \mathrm{M}$ Atglistatin (ATGLi) (Cayman Chemical, cat\# 15284) for 24 hours, or 50 $415 \mu \mathrm{M}$ Lalistat2 (LALi) (Cayman Chemical, cat\# 25347) for 24 hours.

\section{Analysis of Oxygen Consumption Rates}

418 HCC cells were seeded in Seahorse XF96 microplates at a density of $0.75 \times 10^{4}$ cells/well. The next day, 419 the cellular mitochondrial stress test was performed according to the manufacturer's protocol (1 $\mu \mathrm{m}$ 420 oligomycin; $0.75 \mu \mathrm{m}$ FCCP; $5 \mu \mathrm{M}$ Antimycin A), using the Seahorse XF96 analyzer in the Biophysics 421 Core at the University of Chicago. Briefly, 2X DMEM base media was used to make 1X DMEM 
422 supplemented with $4.5 \mathrm{~g} / \mathrm{L}$ glucose, $2 \mathrm{mM}$ glutamine, and $1 \mathrm{mM}$ sodium pyruvate, with a pH adjusted to

423 7.35. Cells were rinsed with PBS prior to addition of 175uL of 1 X DMEM and the plate was incubated in

424 the absence of $\mathrm{CO}_{2}$ for approximately 1 hour. Alternatively, cells were incubated in substrate-limited

425 growth media for $24 \mathrm{~h}$ and then palmitate was added as the main carbon source according to the

426 manufacturer's protocol in the palmitate oxidation stress kit from Agilent Plc, cat\# 103693-100). Data

427 were normalized by cell number using Hoechst 33342 nuclear counterstain and fluorescence

428 quantification using a microplate reader. Normalized OCR data was then analyzed using Agilent

429 Seahorse Wave software.

431 Measurement of Cell Growth Rate.

432 HCC that stably express HA-BNIP3 or HA-BNIP3W18A or Empty Vector as control were seeded into 433 twelve wells each per condition (8*104 cells per well) on 96 well plates. Cells were treated or not with 434 different drugs (TVB-3664, Etomoxir or LALi) and confluence was measured along the time with the 435 IncuCyte Live-Cell Analysis System (Sartorius) over a 7 day period.

\section{RNA extraction}

438 Cells in a 6-well plate were washed twice with $2 \mathrm{~mL}$ of DPBS followed by addition of $1 \mathrm{~mL}$ Trizol. Wells 439 were incubated for 5 minutes at RT and collected in eppendorf tubes. At this step, samples could be 440 frozen at $-80{ }^{\circ} \mathrm{C}$ or immediately extracted for RNA. For extraction, 200uL of chloroform was added to 441 each sample, followed by vigorous shaking for 15 seconds and incubation for 3 minutes at RT. Tubes 442 were centrifuged at $12,000 x g, 15 \mathrm{~min}$, at $4{ }^{\circ} \mathrm{C}$. The aqueous upper phase $(\sim 400 \mu \mathrm{L})$ was transferred into 443 a fresh tube, followed by addition of 1 volume of $70 \% \mathrm{EtOH}(\sim 400 \mathrm{uL})$ and vigorous shaking. Samples 444 were incubated for 5 minutes and then applied to RNeasy columns. The remainder of the extraction was 445 performed according to the RNeasy Mini kit protocol (Qiagen) and included on-column DNasel digestion. 446 RNA was eluted in $50 \mu \mathrm{L}$ of RNase-free water, concentrations were measured using the NanoDrop 447 Spectrophotometer, and samples were stored at $-80^{\circ} \mathrm{C}$.

\section{Quantitative PCR}

450 To make cDNA, 1-2 $\mu \mathrm{g}$ of RNA was reverse transcribed using the High Capacity RNA-to-cDNA kit 451 (Applied Biosystems). The concentration of cDNA was measured by NanoDrop and samples were stored 452 at $-20^{\circ} \mathrm{C}$. For gene expression analysis, we performed quantitative real-time PCR on 250 ng of cDNA 
453 per sample using Taqman gene-specific fluorogenic probes (Applied Biosystems/Thermo Fisher).

454 Primers used for qPCR included in this manuscript are as follows:

455 Genomic copy number (Mt:Nuc gDNA):

456 Cyba: Mm00241140_cn

457 Ndufa1: Mm00526370_cn

458 Hbb-bh1: Mm00216612_cn

459

460 Gene expression:

461 Bnip3: Mm01275601_g1

462 Fasn: Mm01253292_m1

463 Acaca:Mm01304277_m1

464 Acly: Mm00652520_m1

465 Scd1: Mm00772290_m1

466 Cpt1a: Mm00550438_m1

467 Cpt2: Mm00487205_m1

468 Acadm: Mm01323360_g1

469 Acadl: Mm00599660 m1

470 Rps12: Mm03030276_g1

471

\section{Immunohistochemistry \& Oil Red O staining}

473 Immunohistochemistry on mouse liver sections was carried out as described previously ${ }^{22}$ using heat 474 denaturation in citrate buffer $\mathrm{pH} 6.0$ to expose epitope. Stained slides were digitized using an Allied Vision 475 Technologies Stingray F146C color slide scanner and quantified using the Spectrum Plus Image analysis 476 software (Aperio). Antibodies were used in IHC as follows: Ki67 (Labvision, cat\# RM9106), $\alpha-B N I P 3$ 477 (Sigma Prestige, HPA003015) 1:100; $\alpha$-FASN (Cell Signaling Technology, cat\# 3180) 1: 100; $\alpha-A C A C A$ 478 (Cell Signaling Technology, cat\# 3676) 1: 200. Oil Red O staining was performed on frozen liver sections 479 that were warmed to room temperature, fixed for 10 minutes in cold $10 \%$ neutral buffered formalin and 480 allowed to air dry. Slides were incubated in propylene glycol for 3 minutes and then in Oil Red 481 O/propylene glycol solution for 10 minutes, followed by 3 minutes in $85 \%$ propylene glycol and washing 482 three times in water. Oil Red O reagents were obtained from Newcomer Supply (Cat.\# 9119A). Oil Red 483 O stained sections were then counterstained in hematoxylin and mounted using Vectashield (Vector 484 Laboratories). Oil red $\mathrm{O}$ droplets were quantified in the red channel following deconvolution and 485 thresholding, using Image $\mathrm{J}(\mathrm{NIH})$.

\section{Protein extraction}

488 For harvesting of cells, plates were washed in ice-cold DPBS followed by scraping in $1 \mathrm{~mL}$ of DPBS 489 containing protease inhibitors (0.5 mM PMSF, $1 \mu \mathrm{g} / \mathrm{mL}$ aprotinin, $1 \mu \mathrm{g} / \mathrm{mL}$ leupeptin, $\left.1 \mathrm{mM} \mathrm{Na}_{3} \mathrm{VO}_{4}\right)$. 490 Cells were pelleted at $3000 x \mathrm{x}$ for 3 minutes at $4^{\circ} \mathrm{C}$ and resuspended in RIPA lysis buffer (10 mM Tris$491 \mathrm{HCl} \mathrm{pH} 8.0,150 \mathrm{mM} \mathrm{NaCl}, 1 \%$ sodium deoxycholate, $0.1 \%$ SDS, $1 \%$ Trition $\mathrm{X}-100)$ containing 
492 protease and phosphatase inhibitors (Roche PhosSTOP inhibitor cocktail tablet). Samples were

493 incubated on ice for 15 minutes with vortexing every 5 minutes, and centrifuged at full speed for 15

494 minutes at $4{ }^{\circ} \mathrm{C}$. The supernatant was transferred to pre-chilled Eppendorf tubes and protein 495 concentration was measured on a NanoDrop spectrophotometer and stored frozen at $-80^{\circ} \mathrm{C}$.

\section{Western blot}

498 Protein samples were denatured by boiling for 5 min with SDS reducing sample buffer ( $400 \mathrm{mM}$ Tris $\mathrm{pH}$ $4996.8,10 \%$ SDS, $500 \mathrm{mM} \beta$-mercaptoethanol) and sample loading dye (60\% glycerol and bromophenol blue). The amount of protein loaded per sample varied depending on the proteins being probed, but typically $75 \mu \mathrm{g}$ was loaded onto SDS-PAGE gels, followed by transfer to nitrocellulose (0.2 $\mu \mathrm{m}$ pore, GE Healthcare) or PVDF (0.45 $\mu \mathrm{m}$ pore, GE Healthcare) membranes. Membranes were blocked in $5 \%$ nonfat milk in TBS/0.05\% Tween (TBST) for 30 minutes at room temperature with shaking and incubated with primary antibodies overnight at $4{ }^{\circ} \mathrm{C}$ on a rocker, in $5 \%$ BSA/TBST for antibodies from Cell Signaling Technology and in 5\% nonfat milk/TBST for all others. The next day, membranes were washed 3 times with TBST and incubated with HRP-conjugated secondary antibody (Dako) in 5\% nonfat milk/TBS-T for 2 hours at room temperature on a shaker. Membranes were washed 3 times in TBS-T and proteins were visualized by chemiluminescence and exposure on X-ray film. Antibodies for western blots were used as follows: $\alpha-H A$ (Cell Signaling 3724) 1:2,000, $\alpha$-BNIP3 (Cell Signaling \#3769) 1:500, $\alpha$-Alpha fetoprotein (Santa Cruz sc-15375) 1:500.

\section{Immunofluorescence \& confocal microscopy}

513 For immunofluorescence staining, cells were seeded onto 8 well chambered coverslips (Ibidi \#80826) and grown in HCC growth media. Cells were treated with Bafilomycin $\mathrm{A}_{1}$ (Enzo Cat\#: BML-CM110) at $100 \mathrm{nM}$ for the last $4 \mathrm{hr}$ of the experiment. At experimental endpoint, media was aspirated and wells washed in DPBS followed by fixation in 4\% paraformaldehyde (PFA) (Alfa Aesar cat\#: J61899AP) for 15 minutes at RT, followed by permeabilization in ice-cold $100 \%$ methanol for 10 minutes at $-20^{\circ} \mathrm{C}$. Wells were washed with $0.5 \%$ TBST and blocked in $10 \%$ goat serum in $0.5 \%$ TBST for 30 minutes. Wells were incubated with primary antibodies in $10 \%$ goat serum in $0.5 \%$ TBST overnight at $4^{\circ} \mathrm{C}$. Anti-TOMM20 (Abcam, ab56783, 1:150), anti-LC3B (Cell Signaling, 3868S, 1:200). The next day, wells were washed in $0.5 \%$ TBST for $3 \times 5$ minutes, followed by incubation in appropriate fluorescent secondary antibodies in $10 \%$ goat serum/TBST for 1 hour at RT, protected from light. Wells were washed in $0.5 \%$ TBST for $3 \times 5$ minutes and mounted with lbidi mounting medium with DAPI (Ibidi cat\#: 50011). For BODIPY staining, wells were treated with the relevant drug, if lysosomal visualization was required, cells were treated with LysoTracker Deep Red (Invitrogen cat\#: L12492) at 50nM, 4hr before experimental endpoint. Media was 
526 aspirated and wells washed in DPBS followed by fixation in 4\% (PFA) for 10 minutes at RT. Incubated 527 cells in 0.5ug/ml BODIPY 493/503 (Invitrogen cat\#: D3922) diluted in DPBS for 25 mins. Washed 3x with 528 DPBS and mounted with Ibidi mounting medium with DAPI. All imaging was performed using the Leica 529 TCS SP8 laser scanning confocal microscope in the Integrated Microscopy Core Facility at the University 530 of Chicago. All images were collected using a 63X oil-immersion objective. 10-15 images representative 531 images were obtained for each well.

\section{ImageJ quantification of Lipid Droplets}

534 Quantification of BODIPY positive lipid droplets was performed in FIJI. An ImageJ macro was written to 535 threshold the images using the "MaxEntropy" algorithm for auto-thresholding and to count the lipid 536 droplets in each image. A separate macro was written to quantify the number of nuclei in each image in order to calculate the average number of LDs per cell.

\section{Measurement of Malonyl CoA}

540 Cells were collected from confluent $15 \mathrm{~cm}$ plates, scraped in $1 \mathrm{ml}$ DPBS and immediately frozen at $-20^{\circ} \mathrm{C}$. 541 Malonyl CoA levels were measured using a Mouse malonyl coenzyme A ELISA Kit (MyBiosource cat\# 542 MBS705127) according to manufacturer's instructions. Values were normalized by total protein levels 543 measured using a Brafdord assay protein quantification kit (Bio-Rad cat\# 5000201).

\section{Electron Microscopy}

546 Cells were fixed in situ in 2\% glutaraldehyde/4\% paraformaldehyde for 1 hour at room temperature then 547 gently scraped and pelleted at $900 \mathrm{~g}$. The cell pellet was processed for sectioning and electron 548 microscopy by the Electron Microscopy Core facility at the University of Chicago.

\section{Bioinformatic analysis.}

551 Expression data from dataset GSE84073 was downloaded from the GEO database and BioJupies web 552 application ${ }^{63}$ was used to perform bioinformatic analysis of BNIP3 expression and Lipid-Mitochondrial 553 genes between healthy liver samples (GSM2653819 and GSM2653820) vs HCC samples(GSM2653823 554 and GSM2653824). Linear regression correlation analysis between BNIP3 and FASN, ACLY, 555 PPARGC1A or ACADM was performed using BioVinci (Bioturing, San Diego, CA, USA).

556 Liver hepatocellular carcinoma (LIHC) patient's survival analysis was performed using cBioPortal ${ }^{64,65}$

557 and data from the TCGA PanCancer Atlas ( $\mathrm{n}=372$ patients). LIHC patients were classified into different 558 groups according to the BNIP3 and ACACA median expression value. By Kaplan-Meier method we 
559 obtained the overall survival and comparison between curves were made by using Log-Rank test. Data

560 obtained from cBioPortal database does not require ethical approval.

\section{Statistics}

563 All statistical analyses were carried out using GraphPad Prism of raw data. The data were analyzed using 564 one-way or two-way ANOVA with Tukey's post-test with a 95\% confidence interval for data sets involving 565 single parameters or single groups of data. Other datasets involving comparisons amongst multiple 566 groups used Wilcoxon rank sum analyses with a $95 \%$ confidence interval. Data are shown as the mean $567 \pm$ s.e.m. Values of $p \leq 0.05$ are considered significant. ${ }^{*} p \leq 0.05 ;{ }^{* *} p \leq 0.01 ;{ }^{* * *} p \leq 0.001 ;{ }^{* * *} p \leq 0.0001$

\section{Study approval}

570 All work was approved by the University of Chicago Institutional Animal Care and Use Committee under 571 protocols 71155 and 72056. 


\section{Author Contributions.}

573 Maintenance and establishment of mouse model: GB, LED, AT; Tumor analysis and characterization: 574 LED, At, KFM; Cell line generation and characterization: DEB, LED, KFM; qPCR: DB, AT, LED; Western 575 blots: DEB, LED; Fluorescence microscopy: ABH; Seahorse analyses: DEB; Flow cytometry: DEB; 576 Incucyte growth assays: DEB; ELISA assays: $A B H$; Electron microscopy: ABH, KFM; Human 577 TCGA/Bioinformatics analyses: DEB; Study conceptualization: DEB, ABH, KFM; Manuscript preparation: 578 DEB, ABH, KFM; Manuscript review: all authors. 


\section{Acknowledgements.}

580 This work was supported by funding from NIH R01 CA200310 (KFM) and NIH T32 CA009594 (KFM for $581 \mathrm{ABH})$. The authors thank George Kemble and others at 3V-Bioscences for supplying TVB-3664. This 582 work made use of the Human Tissue Resource Center, the Integrated Small Animal Imaging Facility, the 583 Advanced Electron Microscopy Facility and the Digital Light Microscope Facility that are supported by 584 the University of Chicago Comprehensive Cancer Center Support Grant (P30 CA 014599). The authors 585 particularly thank Terri Li, Brian Roman, Yimei Chen and Christine Labno in these core facilities for 586 outstanding technical assistance. 
a

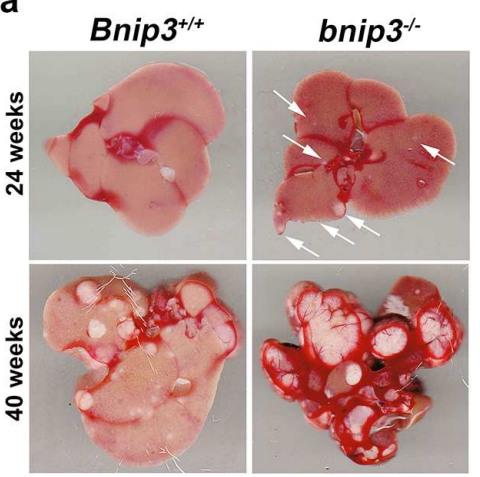

b

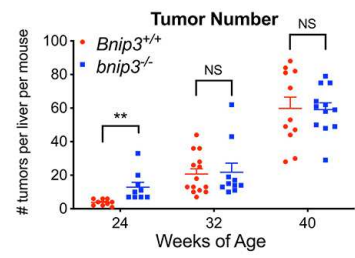

C

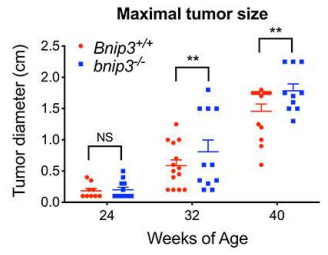

d

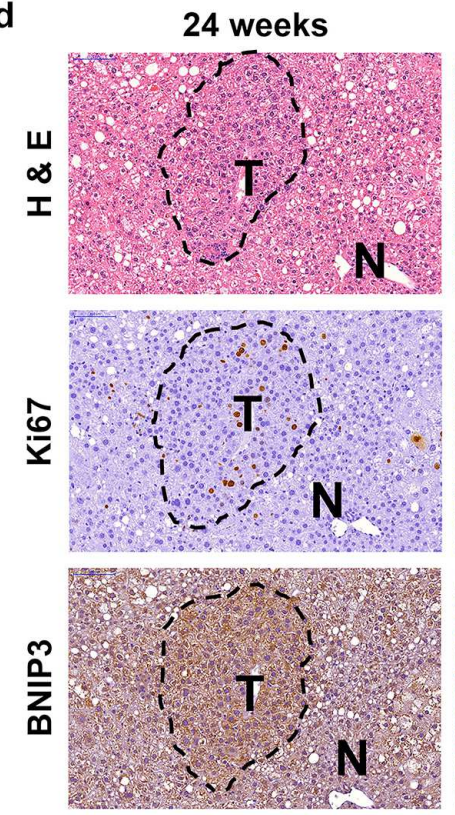

e

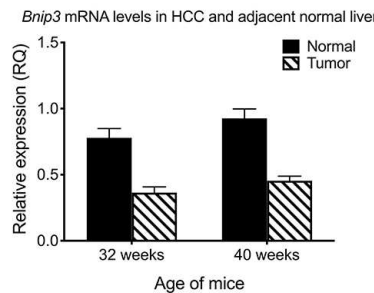

32 weeks
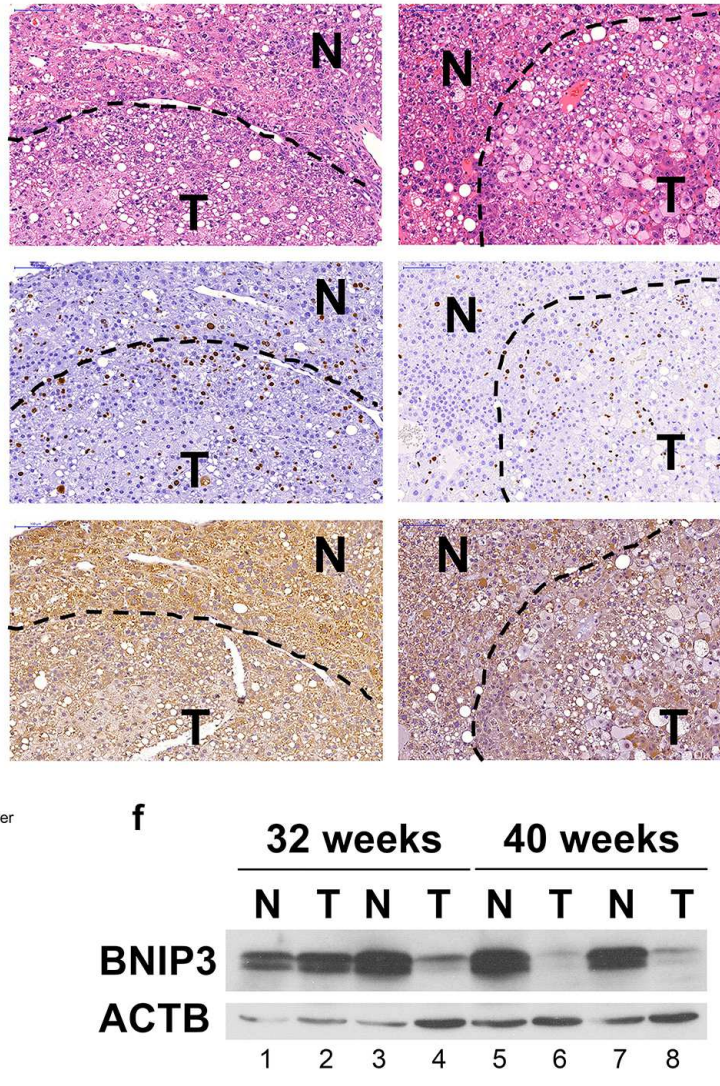

Fig. 1. Loss of BNIP3 promotes HCC initiation and tumor growth. a Representative images of HCC tumors forming in the liver of Bnip3 $3^{+/+}$and bnip $3^{-/-}$mice at 24 weeks and 40 weeks following I.P. injection with $25 \mathrm{mg} / \mathrm{kg}$ DEN. b Graph of tumor number forming in the liver of Bnip3 $3^{+/+}$(red) and bnip3 $3^{-/}$(blue) mice at 24 weeks and 40 weeks following I.P. injection with $25 \mathrm{mg} / \mathrm{kg}$ DEN

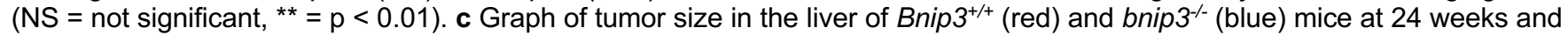
40 weeks following I.P. injection with $25 \mathrm{mg} / \mathrm{kg}$ DEN (NS = not significant, ${ }^{* *}=p<0.01$ ). d Liver sections from Bnip3 ${ }^{+/+}$and bnip $3^{-/}$mice at 24 weeks, 32 weeks and 40 weeks following I.P. injection with $25 \mathrm{mg} / \mathrm{kg}$ DEN, stained with hematoxylin \& eosin (top row), Ki67 (middle row) and BNIP3 (bottom row). e qPCR for Bnip3 mRNA isolated from HCC lesions and adjacent normal liver in Bnip3 ${ }^{+/+}$mice at 32 weeks and 40 weeks weeks following I.P. injection with $25 \mathrm{mg} / \mathrm{kg}$ DEN. f Western blot for BNIP3 in protein lysates isolated from HCC tumor lesions (T) and adjacent normal (N) liver in Bnip3 ${ }^{+/+}$mice at 32 weeks and 40 weeks weeks following I.P. injection with $25 \mathrm{mg} / \mathrm{kg}$ DEN. 
a

a

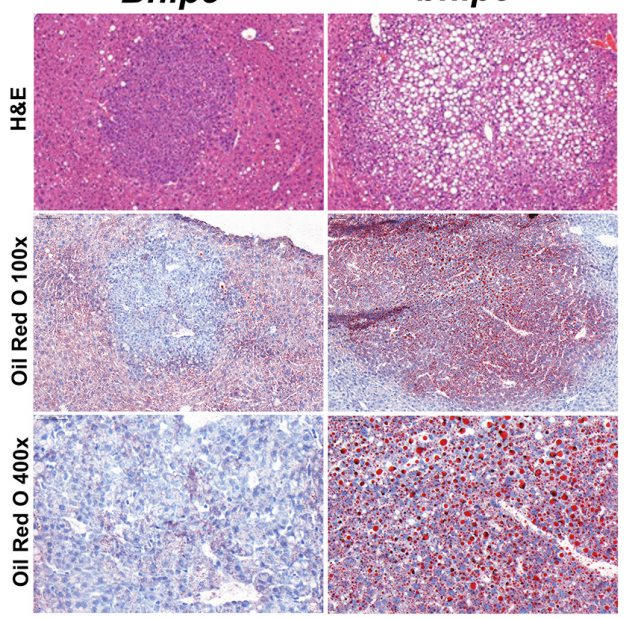

24 weeks

C

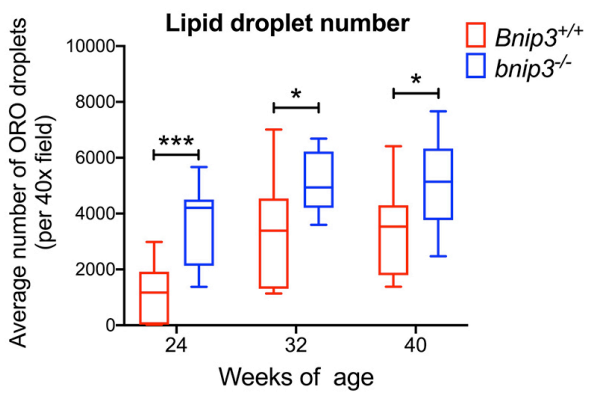

e
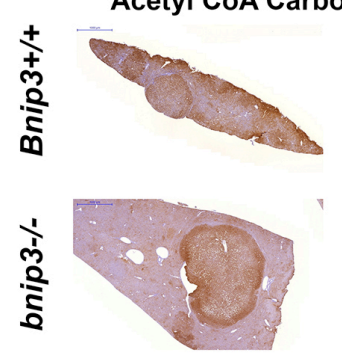

b Bnip3 $3^{+/+} \quad$ bnip3 ${ }^{-/-}$

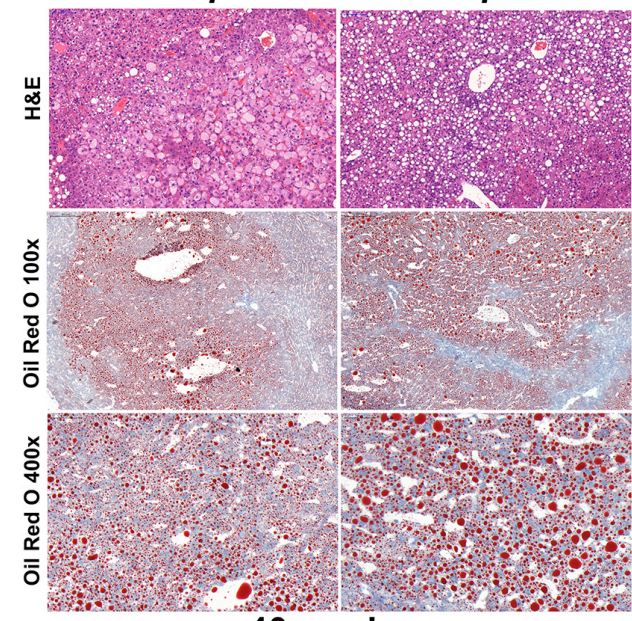

40 weeks

d

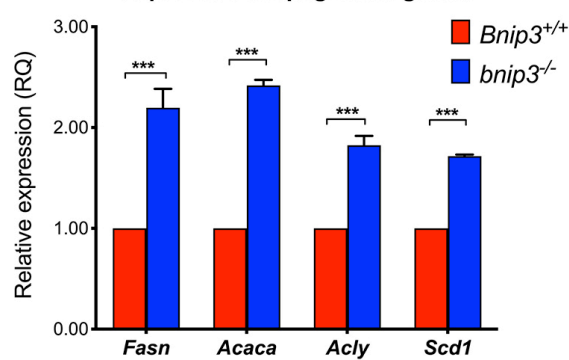

f

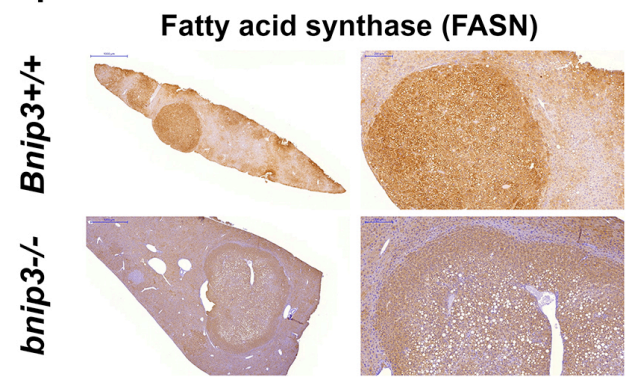

600

601

602

603

604

605

606

607

608

609

610

611

Fig. 2. Lipid increases in HCC when BNIP3 is lost or silenced. a, b Liver sections from Bnip $3^{+/+}$and bnip $3^{-/-}$mice at 24 weeks $(A)$ and 40 weeks (B) following I.P. injection with $25 \mathrm{mg} / \mathrm{kg} \mathrm{DEN}$, stained with hematoxylin \& eosin (top row) or Oil Red O (middle row - 100x magnification, bottom row - 400x magnification). c Graph of Oil Red O-positive lipid droplet number in the liver of Bnip $3^{+/+}$(red) and bnip3 ${ }^{-/-}$(blue) mice at 24 weeks, 32 weeks and 40 weeks following I.P. injection with $25 \mathrm{mg} / \mathrm{kg}$ DEN (NS = not significant, ${ }^{*}=p<0.05,{ }^{* *}=p<0.001$ ). d qPCR for lipogenic genes

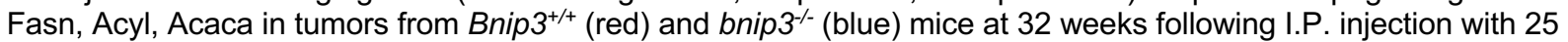
$\mathrm{mg} / \mathrm{kg}$ DEN $\left({ }^{* * *}=\mathrm{p}<0.001\right)$. e Immunohistochemical staining for ACACA in liver sections from Bnip ${ }^{+/+}($red $)$and bnip3 $3^{-/-}$(blue) mice at 24 weeks following I.P. injection with $25 \mathrm{mg} / \mathrm{kg}$ DEN. f Immunohistochemical staining for FASN

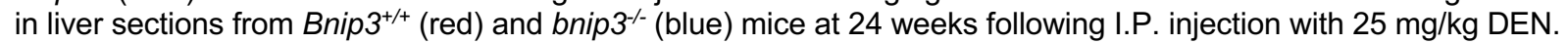


a

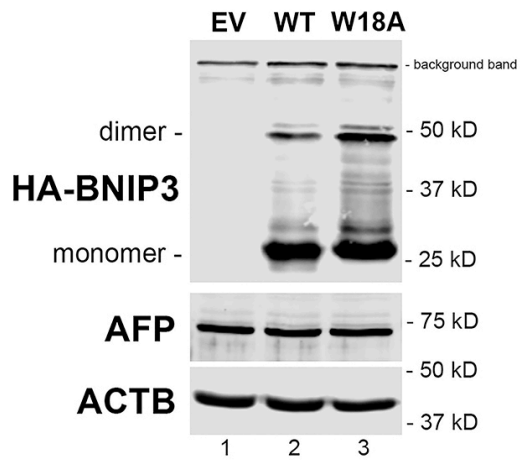

C

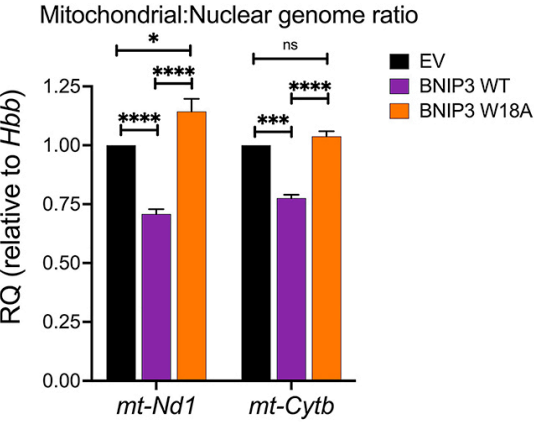

e

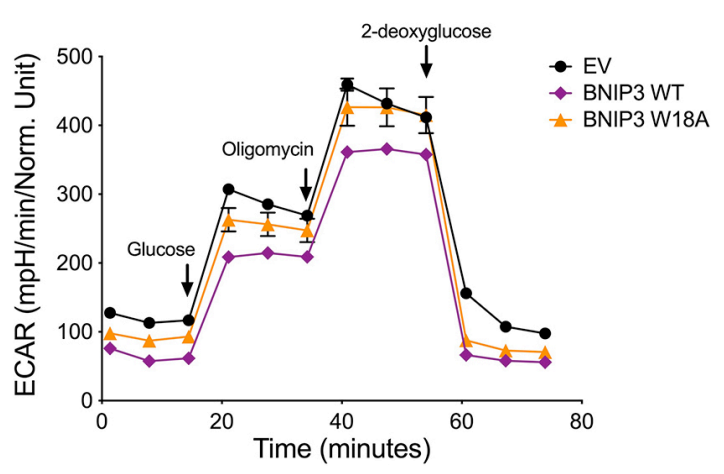

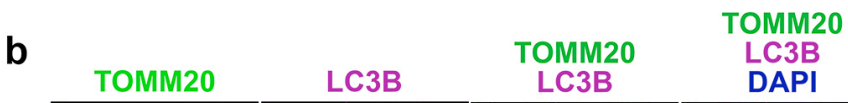

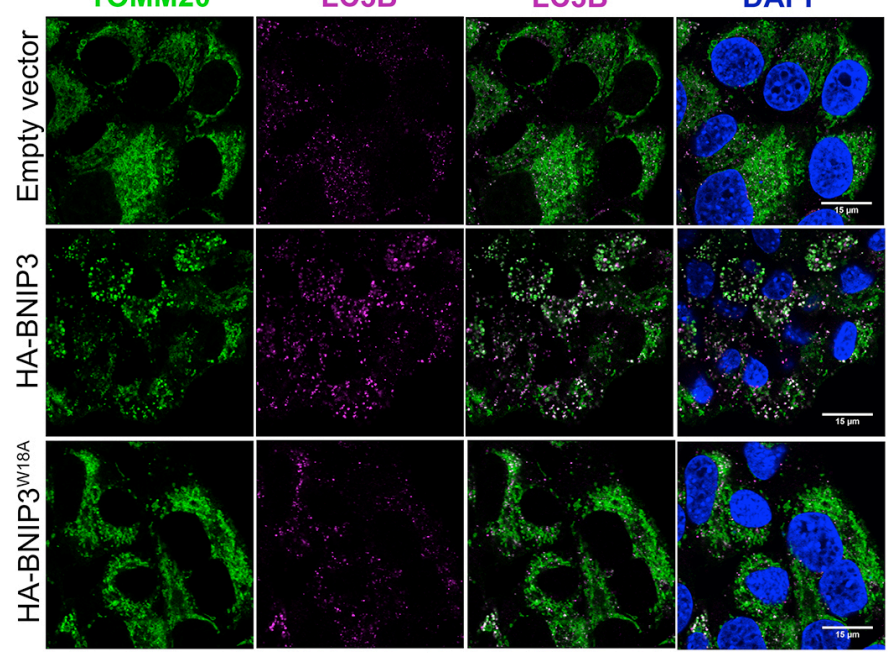

+ Bafilomycin $\mathrm{A}_{1}$

d

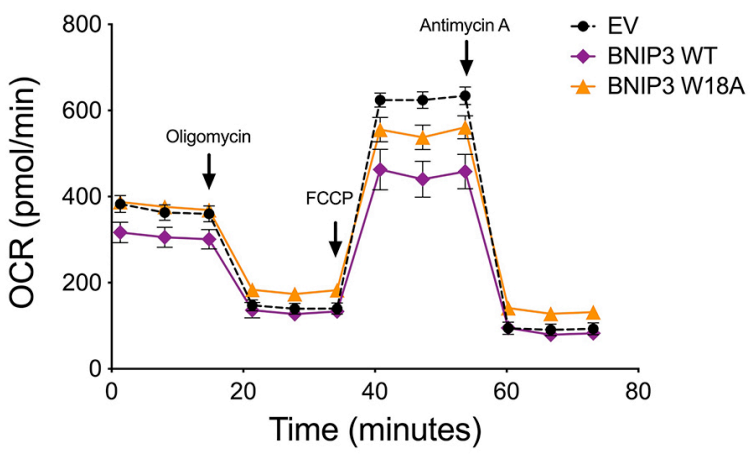

612

614

615

616

617

618

619

620 f

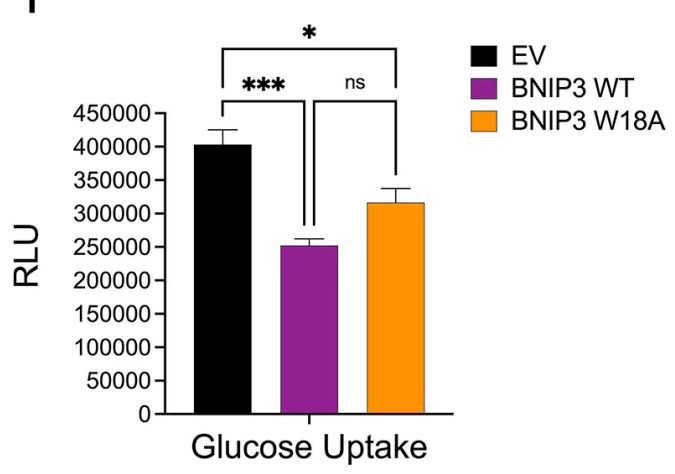

Fig. 3. BNIP3 dependent mitophagy suppresses glucose oxidation, lipid droplet numbers and HCC cell growth. a Western blot for $\alpha-\mathrm{HA}$ and $\alpha \mathrm{FP}$ in protein lysates extracted from bnip $3^{--} \mathrm{HCC}$ cells reconstituted with EV, HA-BNIP3WT or HA-BNIP3 ${ }^{W 18 A}$. b Immunofluorescent staining for TOMM20 and LC3 in bnip $3^{-1-} \mathrm{HCC}$ cells reconstituted with EV, HA-BNIP3 ${ }^{W T}$ or HA-BNIP3 ${ }^{\text {W18A }}$ in the presence of $100 \mathrm{nM}$ bafilomycin $\mathrm{A}_{1}$ for 4 hours. $\mathrm{c}$ qPCR for mt:nuc DNA ratio in bnip $3^{-1-} \mathrm{HCC}$ cells reconstituted with EV, HABNIP3 $^{\text {WT }}$ or HA-BNIP3 ${ }^{W 18 A}$ (NS = not significant, $\left.{ }^{* * *}=p<0.001,{ }^{* * * *}=p<0.0001\right)$. d OCR (glucose) in bnip $3^{-/-}$HCC cells reconstituted with EV, HA-BNIP3 ${ }^{W T}$ or HA-BNIP3 ${ }^{\text {W18A }}$. e ECAR by bnip3 $3^{-1} \mathrm{HCC}$ cells reconstituted with EV, HA-BNIP3 ${ }^{\text {WT }}$ or HABNIP3 ${ }^{\text {W18A }}$ in a glycolysis stress test performed on the Seahorse XF96 extracellular flux analyzer. f Glucose uptake by bnip3 ${ }^{-/-}$ HCC cells reconstituted with EV, HA-BNIP3 ${ }^{W T}$ or HA-BNIP3 ${ }^{\text {W18A. }}$. 
a

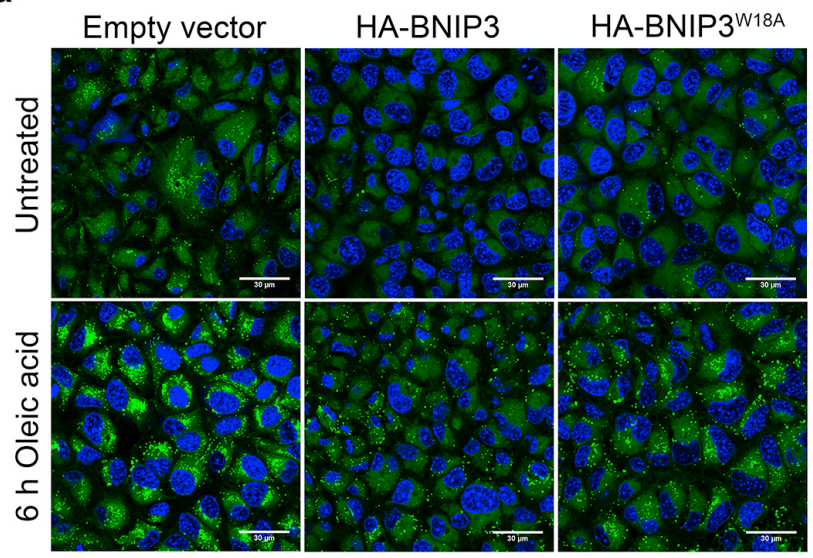

d

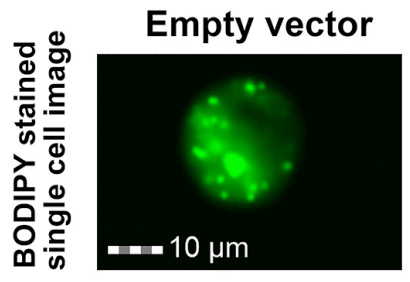

HA-BNIP3

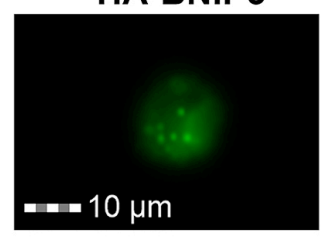

b

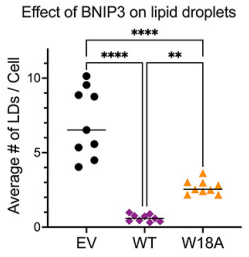

C

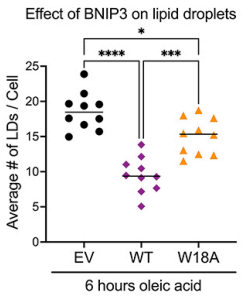

HA-BNIP3 ${ }^{\text {W18A }}$

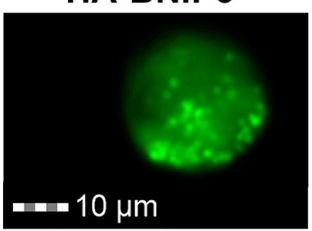

e

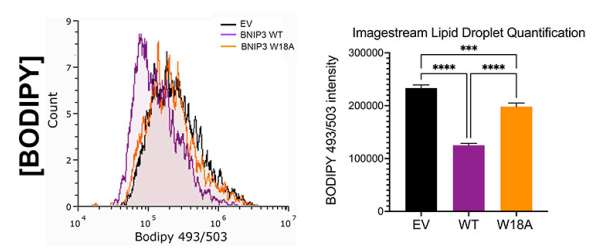

g

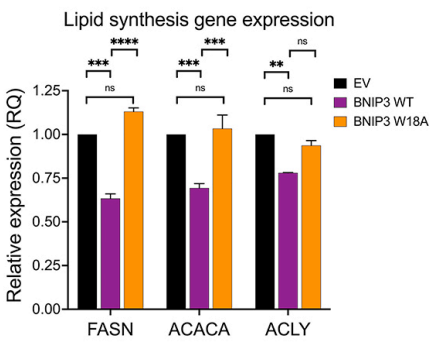

f

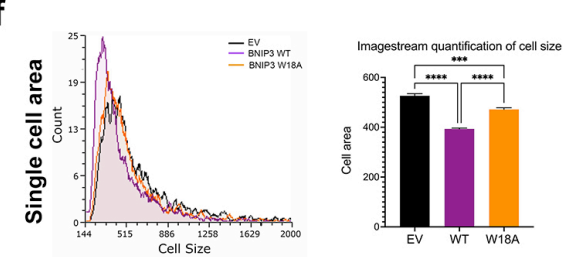

h

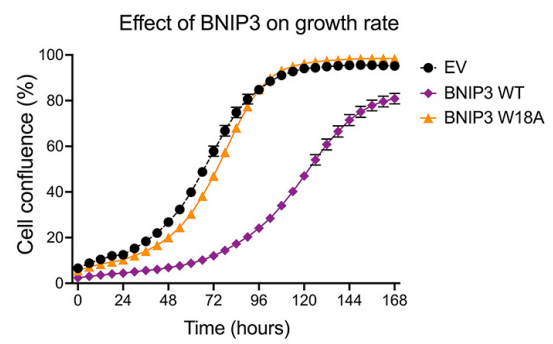

Fig. 4. BNIP3 dependent mitophagy suppresses glucose oxidation, lipid droplet numbers and HCC cell growth. a Lipid droplet number (BODIPY 493/503) in bnip3 $3^{-/}$HCC cells reconstituted with EV, HA-BNIP3WT or HA-BNIP3W18A +/- oleic acid. b Graph of BODIPY 493/503 staining in bnip3 $3^{-/}$HCC cells reconstituted with EV, HA-BNIP3WT or HA-BNIP3 ${ }^{W 18 A}\left({ }^{* * *}, p<0.001 ;{ }^{* * * *}, p<\right.$ 0.0001). c Graph of BODIPY 493/503 staining in bnip3 $3^{--}$HCC cells reconstituted with EV, HA-BNIP3WT or HA-BNIP3W18A + oleic acid $\left(^{* * *}, \mathrm{p}<0.001 ;{ }^{* * *}, \mathrm{p}<0.0001\right)$. d Imagestream analysis of bnip3 $3^{--} \mathrm{HCC}$ cells reconstituted with EV, HA-BNIP3WT or HABNIP3 ${ }^{W 18 A}$ following BODIPY 493/503 staining. e Quantification of BODIPY 493/503 Imagestream analysis of bnip3 ${ }^{-/-} \mathrm{HCC}$ cells reconstituted with EV, HA-BNIP3 ${ }^{W T}$ or HA-BNIP3 ${ }^{W 18 A}\left({ }^{* *}, p<0.001,{ }^{* * *}, p<0.0001\right)$. ${ }^{*}$ Quantification of cell size generated by Imagestream analysis of bnip $3^{--} \mathrm{HCC}$ cells reconstituted with EV, HA-BNIP3 ${ }^{W T}$ or HA-BNIP3 ${ }^{W 18 A}\left({ }^{* * *}, p<0.001,{ }^{* * * *}, p<0.0001\right)$. g qPCR for lipid synthesis genes (FASN, ACACA, ACLY) in bnip3 ${ }^{-/-} \mathrm{HCC}$ cells reconstituted with EV, HA-BNIP3 ${ }^{W T}$ or HA$\mathrm{BNIP}^{\mathrm{W} 18 \mathrm{~A}}$ (NS $=$ not significant, $\left.{ }^{* * *}=\mathrm{p}<0.001,{ }^{* \star * *}=\mathrm{p}<0.0001\right)$. $\mathbf{h}$ Rate of cell growth determined by IncuCyte of $b n i p 3^{-/-} \mathrm{HCC}$ cells reconstituted with EV, HA-BNIP3 ${ }^{\text {WT }}$ or HA-BNIP3 ${ }^{\text {W18A. }}$. 


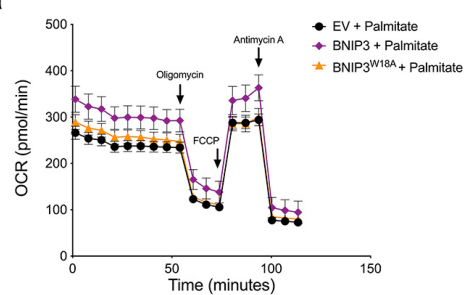

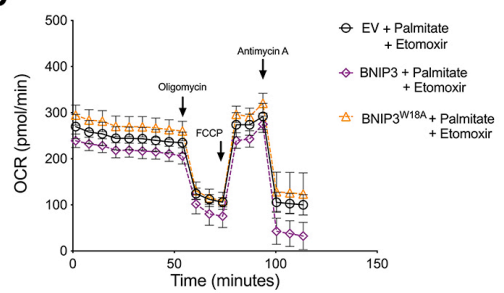

C

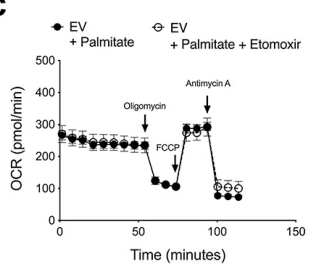

f
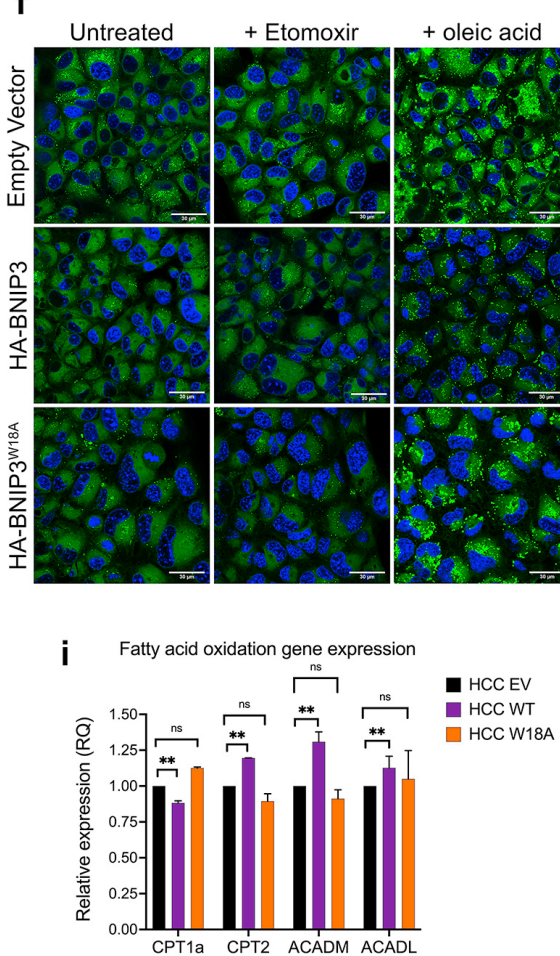

d
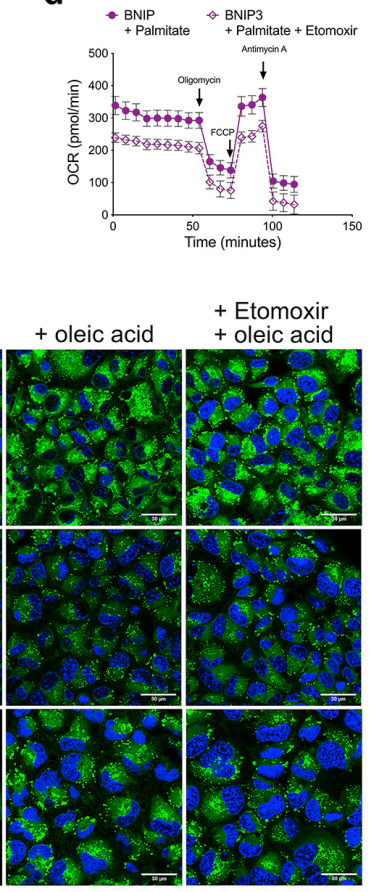

e

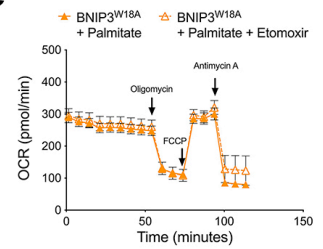

g

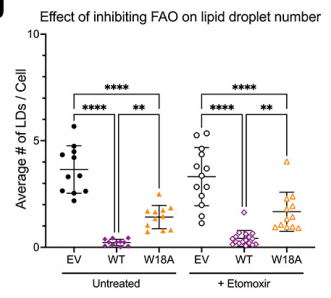

h

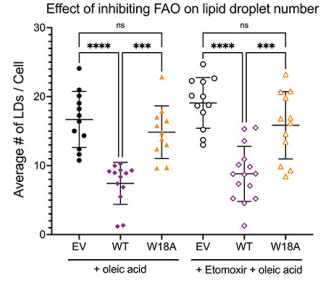

j

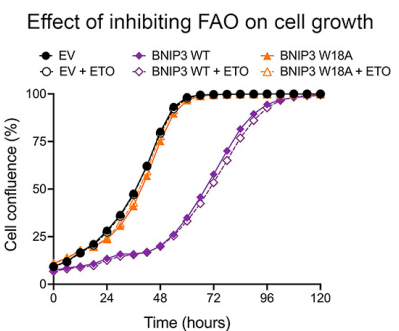

Fig. 5. BNIP3 promotes fatty acid oxidation but this does not explain effects on cell growth. a OCR (palmitate) in bnip $3^{-/-} \mathrm{HCC}$ cells reconstituted with EV, HA-BNIP3 ${ }^{W T}$ or HA-BNIP3 ${ }^{W 18 A}$. b OCR (palmitate) in bnip3 $3^{-/} \mathrm{HCC}$ cells reconstituted with EV, HA-

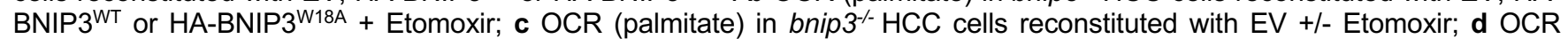
(palmitate) in bnip3 ${ }^{-/-}$HCC cells reconstituted with BNIP3WT +/- Etomoxir; e OCR (palmitate) in bnip3 $3^{-/} \mathrm{HCC}^{-1}$ cells reconstituted with BNIP3 ${ }^{\mathrm{W} 18 \mathrm{~A}}+/$ - Etomoxir; f Fluorescent microscopy imaging of BODIPY 493/503 staining in bnip3 ${ }^{-/} \mathrm{HCC}$ cells reconstituted

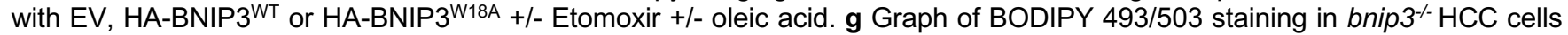
reconstituted with EV, HA-BNIP3WT or HA-BNIP3W18A +/- Etomoxir (NS = not significant, ${ }^{* * *}=p<0.001,{ }^{* * * *}=p<0.0001$ ). $\mathbf{h}$ Graph of BODIPY 493/503 staining in bnip3 ${ }^{-/-}$HCC cells reconstituted with EV, HA-BNIP3WT or HA-BNIP3 ${ }^{\text {W18A }}+/-$ Etomoxir + oleic acid (NS = not significant, $\left.{ }^{* * *}=p<0.001,{ }^{* * * *}=p<0.0001\right)$. i qPCR for FAO genes CPT1a, CPT2, ACADM, ACADL in bnip3 ${ }^{-/-} \mathrm{HCC}$ cells reconstituted with EV, HA-BNIP3 ${ }^{\mathrm{WT}}$ or HA-BNIP3W18A (NS = not significant, ${ }^{* *}=p<0.01$ ). $\mathbf{j}$ Rate of cell growth determined by IncuCyte of bnip $3^{-/-} \mathrm{HCC}$ cells reconstituted with EV, HA-BNIP3WT or HA-BNIP3 ${ }^{\text {W18A }}+/-$ Etomoxir 
a

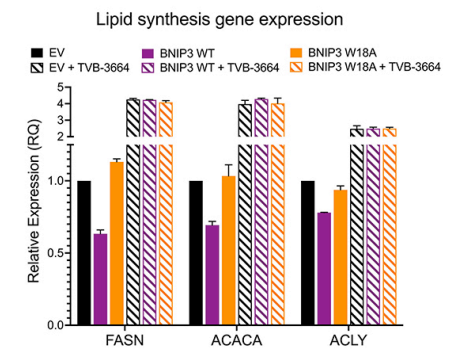

b

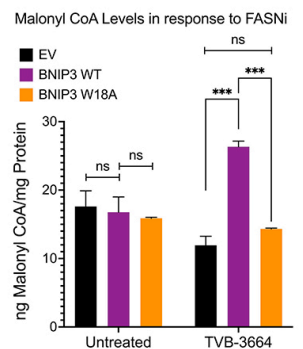

C

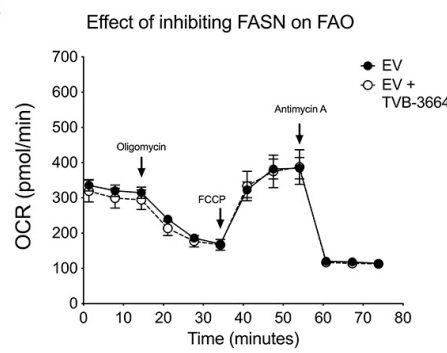

d

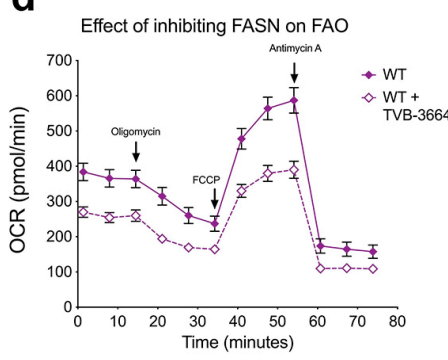

e

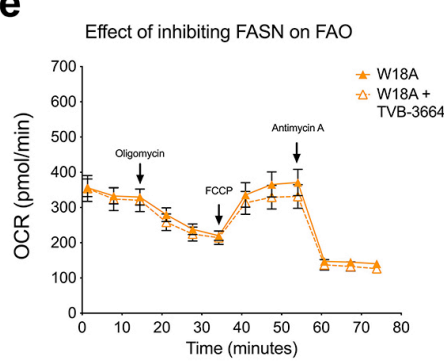

f

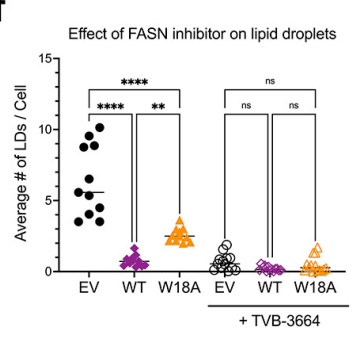

h

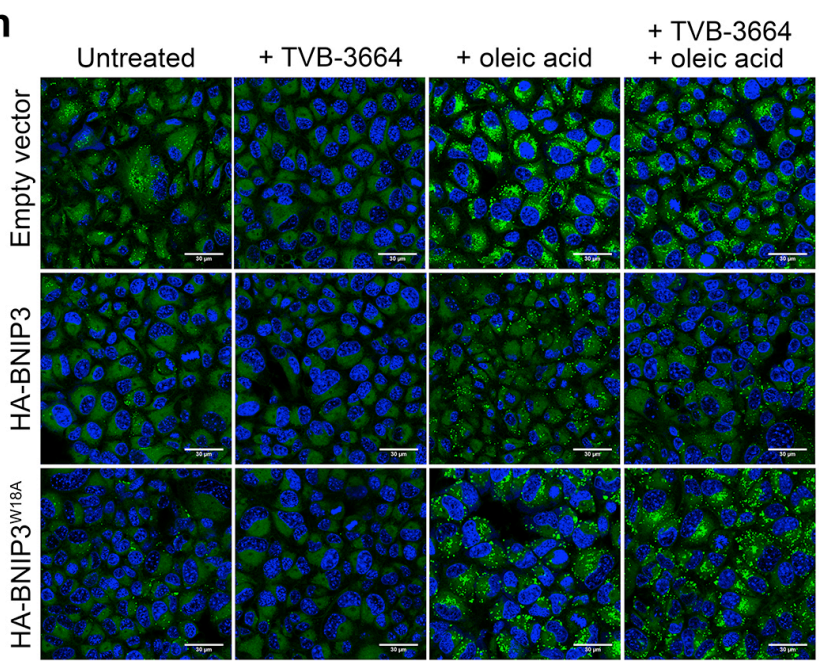

g

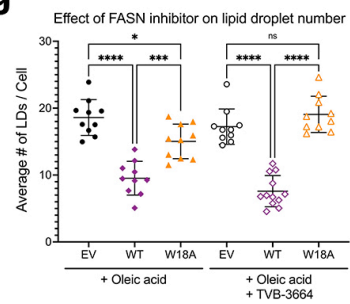

i Effect of FASNi on HCC cell growth

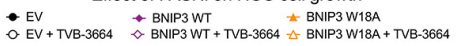

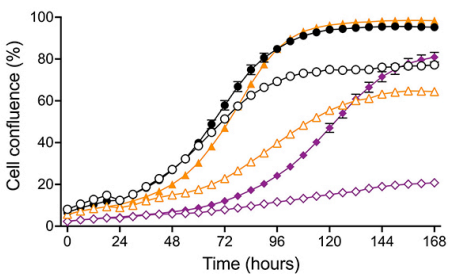

Fig. 6. Inhibiting FASN blocks HCC cell growth but in a BNIP3-independent manner. a qPCR for lipogenesis genes FASN, ACLY, ACACA in bnip3 ${ }^{-/-}$HCC cells reconstituted with EV, HA-BNIP3WT or HA-BNIP3W18A +/- TVB-3664. b Graph of Malonyl CoA levels in bnip $3^{-1}$ HCC cells reconstituted with EV, HA-BNIP3 ${ }^{\mathrm{WT}}$ or HA-BNIP3 ${ }^{\mathrm{W} 18 \mathrm{~A}}+/-$ TVB-3664 $\left(\mathrm{NS}=\right.$ not significant, ${ }^{* * *}=$ $\mathrm{p}<0.001$ ); $\mathbf{c}$ Effect of TVB-3664 on OCR (palmitate) in bnip $3^{-/} \mathrm{HCC}$ cells reconstituted with EV. $\mathbf{d}$ Effect of TVB-3664 on OCR (palmitate) in bnip $3^{--}$HCC cells reconstituted with HA-BNIP3WT; e Effect of TVB-3664 on OCR (palmitate) in bnip $3^{-1}$ HCC cells reconstituted with HA-BNIP3 ${ }^{\text {W18A }} ; \mathbf{f}$ Graph of BODIPY 493/503 staining in bnip3 ${ }^{-/}$HCC cells reconstituted with EV, HA-BNIP3WT or HA-BNIP3 ${ }^{W 18 A}+/-$ TVB (NS = not significant, ${ }^{* *}=p<0.01,{ }^{* * * *}=p<0.0001$ ); $\mathbf{g}$ Graph of BODIPY 493/503 staining in bnip31- $\mathrm{HCC}$ cells reconstituted with EV, HA-BNIP3 ${ }^{\mathrm{WT}}$ or HA-BNIP3 ${ }^{\mathrm{W} 18 \mathrm{~A}}+/$ - Etomoxir + Oleic acid $\left(\mathrm{NS}=\right.$ not significant, ${ }^{*}=p<0.05$, $\left.{ }^{* * *}=p<0.001{ }^{* * * *}=p<0.0001\right)$; h Fluorescent microscopy imaging of BODIPY 493/503 staining in bnip $3^{-1-}$ HCC cells reconstituted with EV, HA-BNIP3 ${ }^{\mathrm{WT}}$ or HA-BNIP3 ${ }^{\mathrm{W} 18 \mathrm{~A}}+$ +- TVB-3664 +/- oleic acid; i Rate of cell growth determined by IncuCyte of bnip $3^{-1}$ HCC cells reconstituted with EV, HA-BNIP3 ${ }^{W T}$ or HA-BNIP3W18A +/- TVB-3664. 


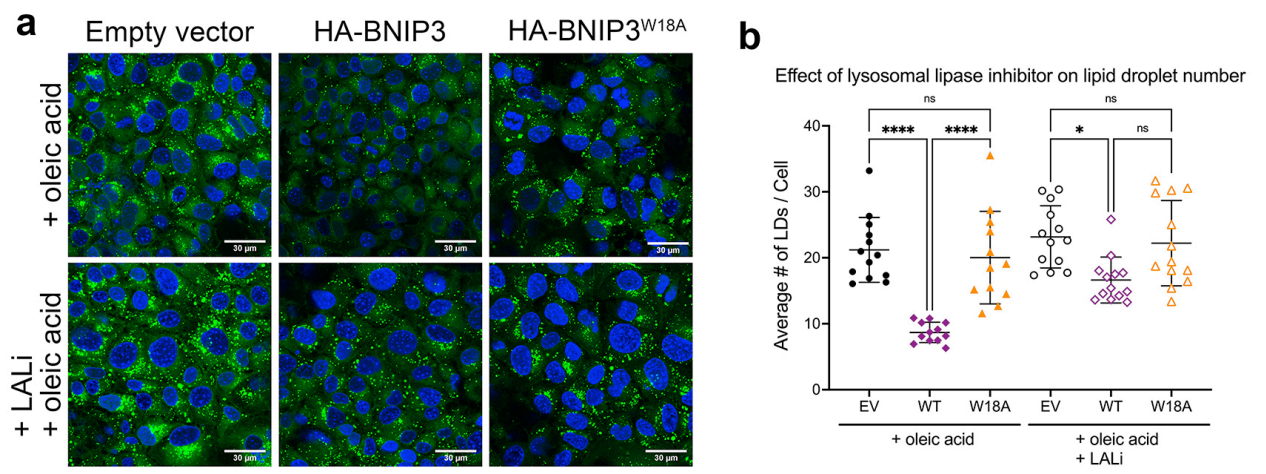

C

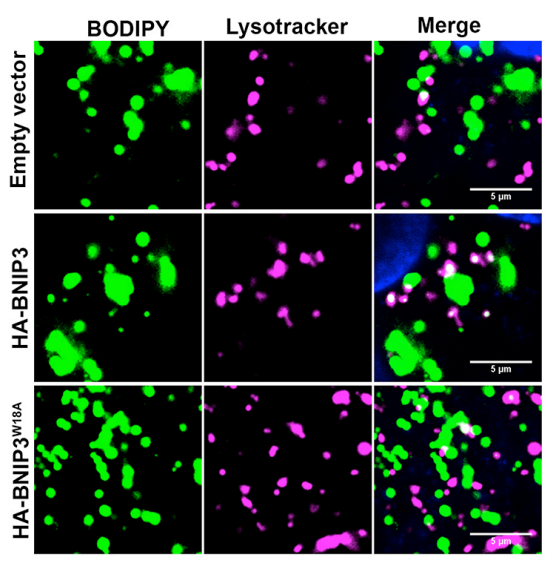

d

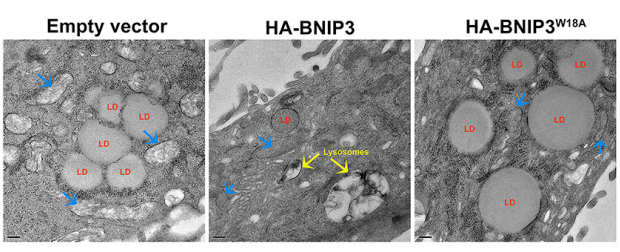

e

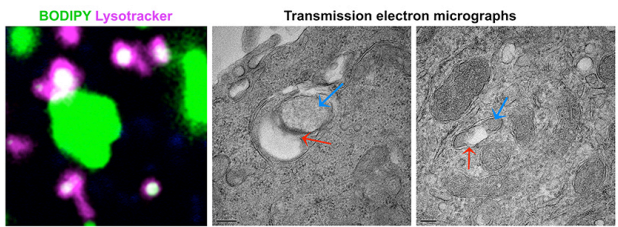

f

g
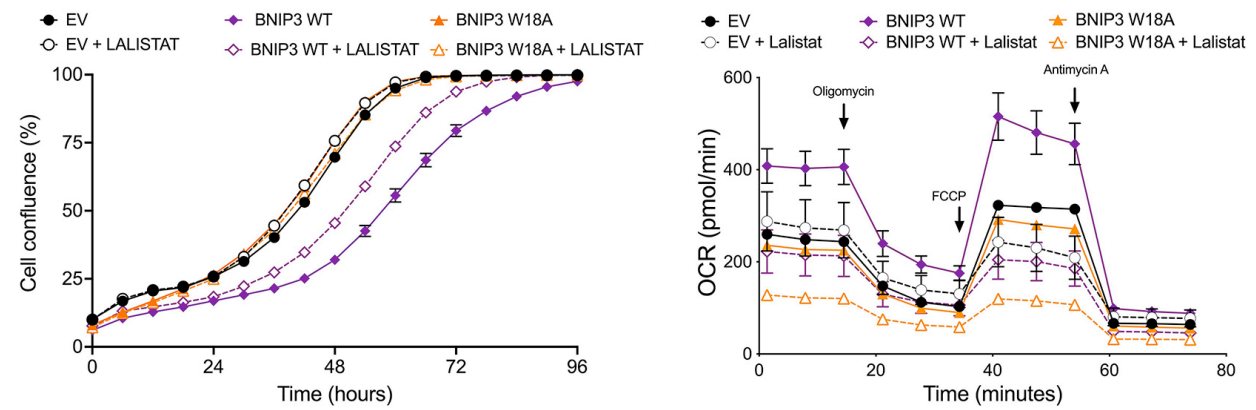

661 Fig. 7. BNIP3 promotes lipophagy and inhibiting lysosomal lipase limits HCC cell growth. a Fluorescent microscopy imaging of 662 BODIPY 493/503 staining in bnip3 $3^{-/-}$HCC cells reconstituted with EV, HA-BNIP3 ${ }^{\text {WT }}$ or HA-BNIP3 ${ }^{\text {W18A }}+/-$ LALi + oleic acid; b 663 Graph of BODIPY 493/503 staining in bnip3 $3^{-/-}$HCC cells reconstituted with EV, HA-BNIP3 ${ }^{\text {WT }}$ or HA-BNIP3 ${ }^{\text {W18A }}+/-$ LALi + Oleic 664 acid (NS = not significant, ${ }^{*}=p<0.05,{ }^{* *}=p<0.01,{ }^{* * * *}=p<0.0001$ ); c Fluorescent microscopy imaging of BODIPY 493/503 665 and Lysotracker stained bnip $3^{-/-}$HCC cells reconstituted with EV, HA-BNIP3 ${ }^{\mathrm{WT}}$ or HA-BNIP3 ${ }^{\mathrm{W} 18 \mathrm{~A}}+$ LALi + oleic acid; d TEM on 666 bnip $3^{--}$HCC cells reconstituted with EV, HA-BNIP3WT or HA-BNIP3 ${ }^{\mathrm{W} 18 \mathrm{~A}}+$ LALi + oleic acid (red: lipid droplets; blue: 667 mitochondria; yellow: lysosomes); e Comparison of fluorescent staining of BODIPY (green) and Lysotracker (magenta) with TEM 668 in bnip $3^{-/}$HCC cells reconstituted with HA-BNIP3 ${ }^{\text {WT }}$ showing overlap between mitochondria and lipid droplets. f OCR (palmitate) 669 in bnip $3^{-/-} \mathrm{HCC}$ cells reconstituted with EV, HA-BNIP3 ${ }^{\mathrm{WT}}$ or HA-BNIP3 ${ }^{\mathrm{W} 18 \mathrm{~A}}+/-$ LALi; g Rate of cell growth determined by IncuCyte 670 of bnip3 ${ }^{-/-}$HCC cells reconstituted with EV, HA-BNIP3 ${ }^{W T}$ or HA-BNIP3 ${ }^{W 18 A}+/-$ LALi. 
a

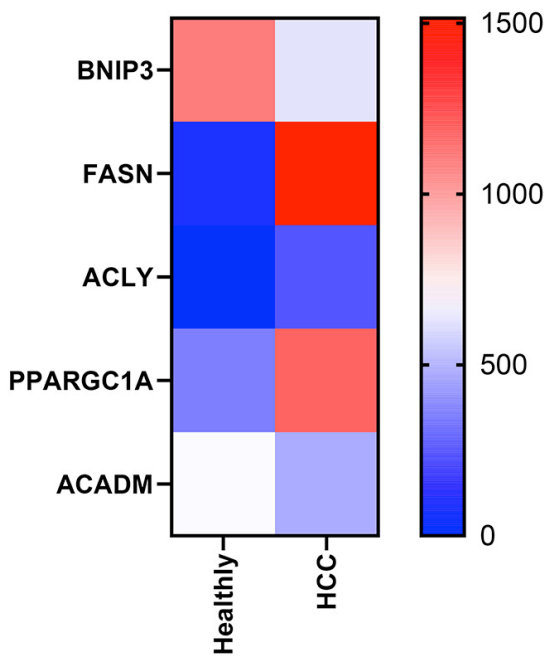

C

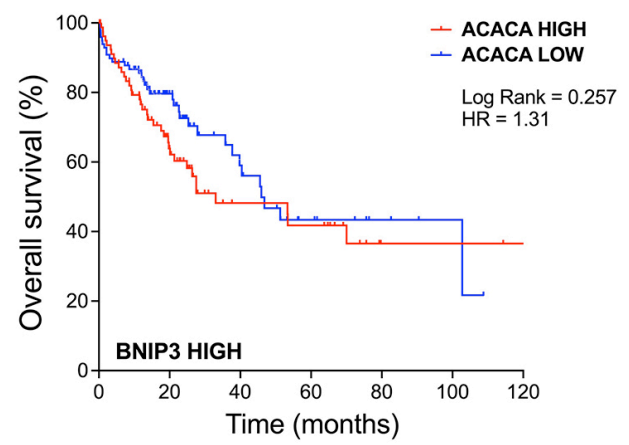

e

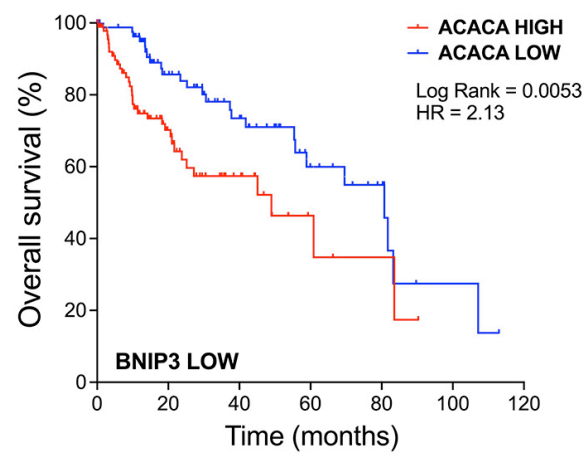

b

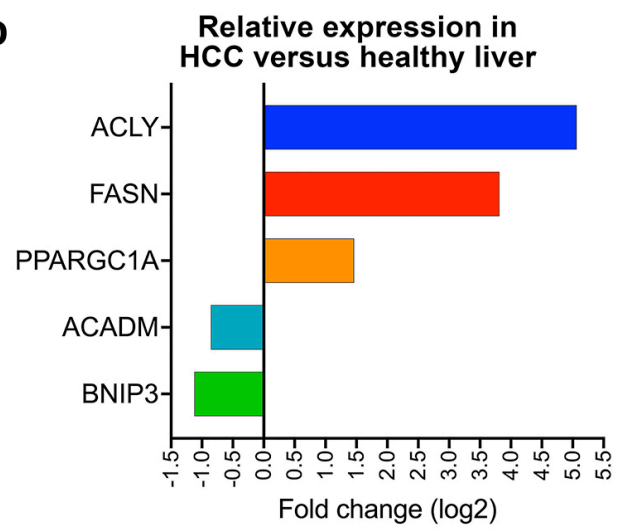

\begin{tabular}{|c|c|c|}
\hline gene symbol & P Value & FDR value \\
\hline BNIP3 & 0.000129505 & 0.000669282 \\
\hline FASN & $2.25 \mathrm{E}-06$ & 0.000196001 \\
\hline ACLY & 0.000161937 & 0.000756413 \\
\hline PPARGC1A & $3.73 \mathrm{E}-05$ & 0.000368858 \\
\hline ACADM & 0.000258373 & 0.000992392 \\
\hline
\end{tabular}

d ACACA LOW
TCGA-DD-AAVR
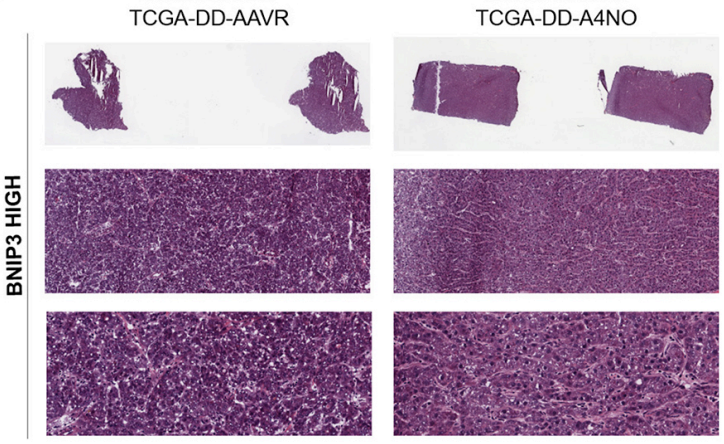

f

f $\begin{array}{r}\text { ACACA LOW } \\ \text { TCGA-DD-A118 }\end{array}$

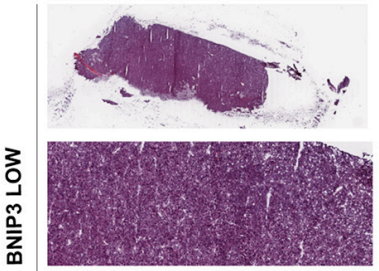

ACACA HIGH
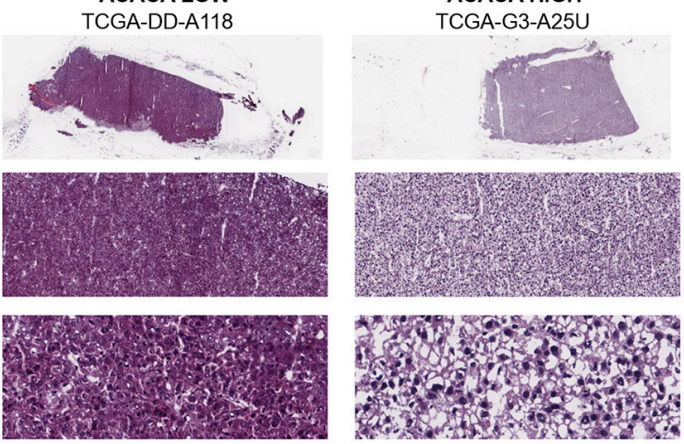

Fig. 8. Low BNIP3 expression in human HCC correlates with high expression of lipogenic genes and poor survival. a Comparison of relative expression of BNIP3, FASN, ACLY, PPARGC1A, ACADM in RNA-Seq analysis of healthy liver and HCC. b Comparison of the relative effect on levels of BNIP3, FASN and ACLY in healthy liver and HCC. c Overall HCC survival data obtained from the TCGA database for tumor showing BNIP3 ${ }^{H I G H}$ expression with either ACACA ${ }^{\text {LOW }}$ or ACACA ${ }^{\text {HIGH }}$ expression. d Hematoxylin \& eosin stained sections of human BNIP3 ${ }^{H I G H}$-expressing HCC with either ACACA ${ }^{\text {LOW }}$ or ACACA ${ }^{H I G H}$ expressing HCC. e Overall HCC survival data obtained from the TCGA database for tumor showing BNIP3 ${ }^{\text {LOW }}$ expression with either ACACA ${ }^{\text {LOW }}$ or ACACA $A^{H I G H}$ expression. $f$ Hematoxylin \& eosin stained sections of human BNIP3 ${ }^{\text {LOW }}$-expressing HCC with either 683 


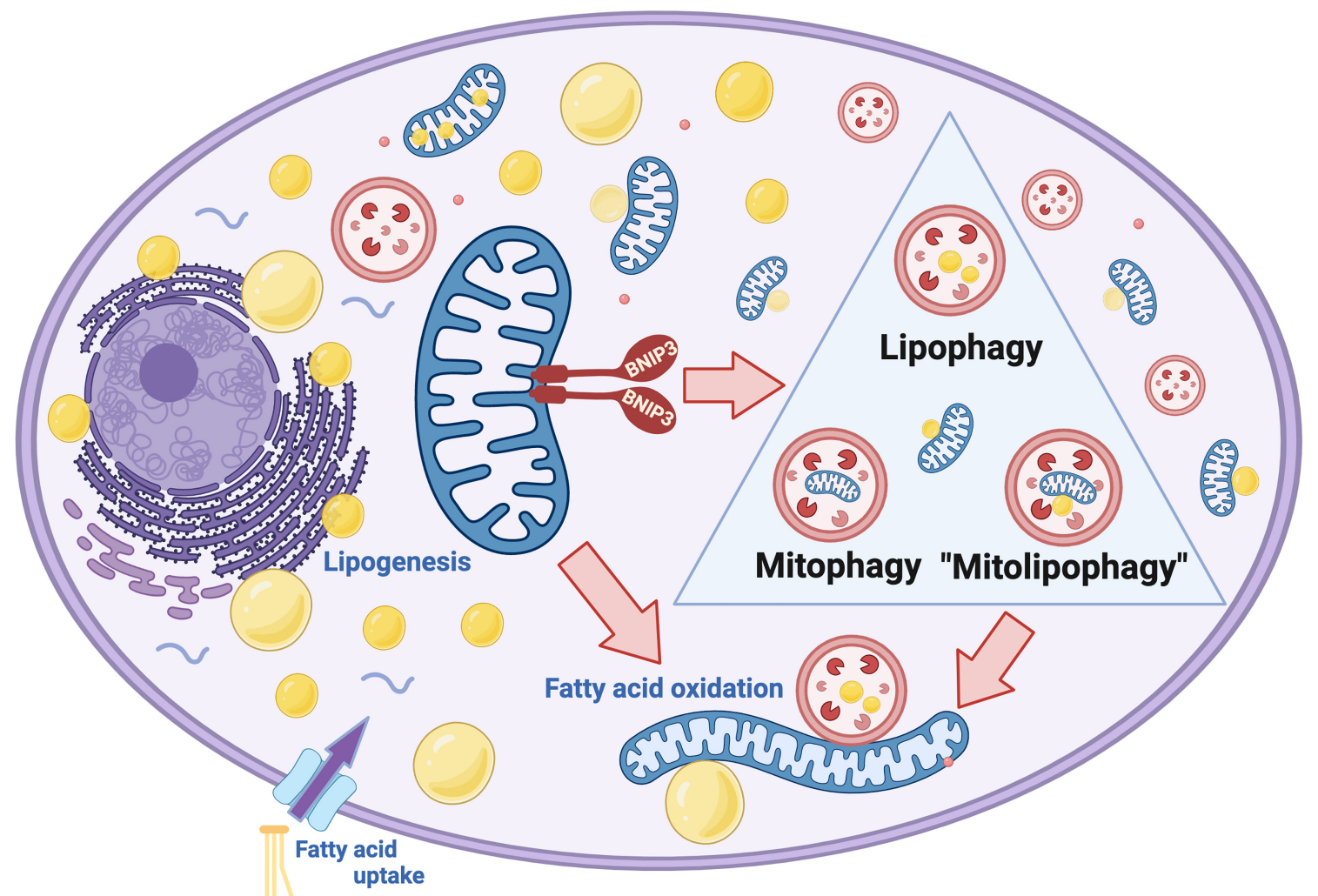

Fig. 9. Diagram summarizing effects of BNIP3 on lipid metabolism in HCC cells.

BNIP3 promotes mitochondrial turnover at the autolysosome (mitophagy) and here we propose that BNIP3-dependent mitophagy also promotes lysosomal turnover of mitochondrial-associated lipid droplets in a process termed "mitolipophagy", a hybrid form of mitophagy and lipophagy (selective turnover of lipid droplets at the autolysosome). Fatty acids liberated from lipid droplets do fuel fatty acid oxidation in HCC cells but this does not explain the growth suppressive effects of BNIP3 in HCC. Rather we propose that lipid droplets promote tumor growth in other ways, such as serving as a reservoir for lipids to promote plasma membrane growth and organelle biogenesis. 
696

697

698

699

700

701

702

703

704

705

706

707

708

709

710

711

712

713

714

715

716

717

718

719

720

721

722

723

724

725

726

727

728

729

730

731

732

733

734

735

736

737

738

739

740

741

742

743

744

\section{References}

1 Cusi, K. Role of obesity and lipotoxicity in the development of nonalcoholic steatohepatitis: pathophysiology and clinical implications. Gastroenterology 142, 711-725.e716, doi:10.1053/j.gastro.2012.02.003 (2012).

2 Sharma, M. et al. The Riddle of Nonalcoholic Fatty Liver Disease: Progression From Nonalcoholic Fatty Liver to Nonalcoholic Steatohepatitis. Journal of clinical and experimental hepatology 5, 147-158, doi:10.1016/j.jceh.2015.02.002 (2015).

3 Woo Baidal, J. A. \& Lavine, J. E. The intersection of nonalcoholic fatty liver disease and obesity. Science translational medicine 8, 323rv321, doi:10.1126/scitranslmed.aad8390 (2016).

4 Samuel, V. T. \& Shulman, G. I. Nonalcoholic Fatty Liver Disease as a Nexus of Metabolic and Hepatic Diseases. Cell Metab 27, 22-41, doi:10.1016/j.cmet.2017.08.002 (2018).

5 Baffy, G., Brunt, E. M. \& Caldwell, S. H. Hepatocellular carcinoma in non-alcoholic fatty liver disease: an emerging menace. Journal of hepatology 56, 1384-1391, doi:10.1016/j.jhep.2011.10.027 (2012).

6 Estes, C., Razavi, H., Loomba, R., Younossi, Z. \& Sanyal, A. J. Modeling the epidemic of nonalcoholic fatty liver disease demonstrates an exponential increase in burden of disease. Hepatology 67, 123-133, doi:10.1002/hep.29466 (2018).

7 Kanwal, F. et al. Risk of Hepatocellular Cancer in Patients With Non-Alcoholic Fatty Liver Disease. Gastroenterology 155, 1828-1837.e1822, doi:10.1053/j.gastro.2018.08.024 (2018).

8 Younossi, Z. et al. Nonalcoholic Steatohepatitis Is the Fastest Growing Cause of Hepatocellular Carcinoma in Liver Transplant Candidates. Clin Gastroenterol Hepatol 17, 748-755.e743, doi:10.1016/j.cgh.2018.05.057 (2019).

9 Younossi, Z. M. et al. Epidemiology of chronic liver diseases in the USA in the past three decades. Gut 69, 564-568, doi:10.1136/gutjnl-2019-318813 (2020).

10 Loomba, R., Friedman, S. L. \& Shulman, G. I. Mechanisms and disease consequences of nonalcoholic fatty liver disease. Cell 184, 2537-2564, doi:10.1016/j.cell.2021.04.015 (2021).

11 Calle, E. E., Rodriguez, C., Walker-Thurmond, K. \& Thun, M. J. Overweight, obesity and mortality from cancer in a prospectively studied cohort of US adults. N. Engl.J.Med. 348, 1625-1638 (2003).

12 Larsson, S. C. \& Wolk, A. Overweight, obesity and risk of liver cancer: a meta-analysis of cohort studies. Br. J. Cancer 97, 1005-1008 (2007).

13 Park, E. J. et al. Dietary and genetic obesity promote liver inflammation and tumorigenesis by enhancing IL-6 and TNF expression. Cell 140, 197-208 (2010).

14 Umemura, A. et al. Liver damage, inflammation, and enhanced tumorigenesis after persistent mTORC1 inhibition. Cell Metab 20, 133-144, doi:10.1016/j.cmet.2014.05.001 (2014).

$15 \mathrm{Li}, \mathrm{X}$. et al. The immunological and metabolic landscape in primary and metastatic liver cancer. Nat Rev Cancer 21, 541-557, doi:10.1038/s41568-021-00383-9 (2021).

16 Brunt, E. M. Nonalcoholic steatohepatitis. Semin. Liver Dis. 24, 3-20 (2004).

17 Begriche, K., Igoudjil, A., Pessayre, D. \& Fromenty, B. Mitochondrial dysfunction in NASH: causes, consequences and possible means to prevent it. Mitochondrion 6, 1-28 (2006).

18 Mansouri, A., Gattolliat, C. H. \& Asselah, T. Mitochondrial Dysfunction and Signaling in Chronic Liver Diseases. Gastroenterology 155, 629-647, doi:10.1053/j.gastro.2018.06.083 (2018).

19 Font-Burgada, J., Sun, B. \& Karin, M. Obesity and Cancer: The Oil that Feeds the Flame. Cell Metab 23, 48-62, doi:10.1016/j.cmet.2015.12.015 (2016).

20 Macleod, K. F. Mitophagy and Mitochondrial Dysfunction in Cancer. Ann. Rev. Cancer Biol. 4, 41 - 60 (2020).

21 Lee, J. M. et al. Nutrient-sensing nuclear receptors coordinate autophagy. Nature 516, 112-115 (2014). 
22 Springer, M. Z. et al. BNIP3-dependent mitophagy promotes cytosolic localization of LC3B and metabolic homeostasis in the liver. Autophagy, 1-17, doi:10.1080/15548627.2021.1877469 (2021).

23 Verna, L., Whysner, J. \& Williams, G. M. N-nitrosodiethylamine mechanistic data and risk assessment: bioactivation, DNA-adduct formation, mutagenicity, and tumor initiation. Pharmacol Ther 71, 57-81, doi:10.1016/0163-7258(96)00062-9 (1996).

24 Maeda, S., Kamata, H., Luo, J. L., Leffert, H. \& Karin, M. IKKb couples hepatocyte death to cytokine-driven compensatory proliferation that promotes chemical hepatocarcinogenesis. Cell 121, 977-990 (2005).

25 Kitami, T. et al. A chemical screen probing the relationship between mitochondrial content and cell size. PloS ONE 7, e33755 (2012).

26 Miettinen, T. P. \& Björklund, M. Cellular Allometry of Mitochondrial Functionality Establishes the Optimal Cell Size. Dev Cell 39, 370-382, doi:10.1016/j.devcel.2016.09.004 (2016).

27 Snaebjornsson, M. T., Janaki-Raman, S. \& Schulze, A. Greasing the Wheels of the Cancer Machine: The Role of Lipid Metabolism in Cancer. Cell Metab 31, 62-76, doi:10.1016/j.cmet.2019.11.010 (2020).

28 Benjamin, D. I. et al. Diacylglycerol Metabolism and Signaling Is a Driving Force Underlying FASN Inhibitor Sensitivity in Cancer Cells. ACS Chem.Biol. AOP (2015).

29 Shimano, H. \& Sato, R. SREBP-regulated lipid metabolism: convergent physiology - divergent pathophysiology. Nature reviews. Endocrinology 13, 710-730, doi:10.1038/nrendo.2017.91 (2017).

30 Foster, D. W. Malonyl-CoA: the regulator of fatty acid synthesis and oxidation. The Journal of clinical investigation 122, 1958-1959, doi:10.1172/jci63967 (2012).

31 Welte, M. A. Expanding roles for lipid droplets. Current biology : CB 25, R470-481, doi:10.1016/j.cub.2015.04.004 (2015).

32 Singh, R. et al. Autophagy regulates lipid metabolism. Nature 458, 1131-1139 (2009).

33 Schott, M. B. et al. Lipid droplet size directs lipolysis and lipophagy catabolism in hepatocytes. $J$ Cell Biol 218, 3320-3335, doi:10.1083/jcb.201803153 (2019).

34 Schulze, R. J. et al. Direct lysosome-based autophagy of lipid droplets in hepatocytes. Proc Natl Acad Sci U S A 117, 32443-32452, doi:10.1073/pnas.2011442117 (2020).

35 Freyre, C. A. C., Rauher, P. C., Ejsing, C. S. \& Klemm, R. W. MIGA2 Links Mitochondria, the ER, and Lipid Droplets and Promotes De Novo Lipogenesis in Adipocytes. Molecular cell 76, 811825.e814, doi:10.1016/j.molcel.2019.09.011 (2019).

36 Veliova, M., Petcherski, A., Liesa, M. \& Shirihai, O. S. The biology of lipid droplet-bound mitochondria. Seminars in cell \& developmental biology 108, 55-64, doi:10.1016/j.semcdb.2020.04.013 (2020).

37 Benador, I. Y., Veliova, M., Liesa, M. \& Shirihai, O. S. Mitochondria Bound to Lipid Droplets: Where Mitochondrial Dynamics Regulate Lipid Storage and Utilization. Cell Metab 29, 827-835, doi:10.1016/j.cmet.2019.02.011 (2019).

38 Broutier, L. et al. Human primary liver cancer-derived organoid cultures for disease modeling and drug screening. Nature medicine 23, 1424-1435, doi:10.1038/nm.4438 (2017).

39 Yahagi, N. et al. Co-ordinate activation of lipogenic enzymes in hepatocellular carcinoma. Eur J Cancer 41, 1316-1322, doi:10.1016/j.ejca.2004.12.037 (2005).

40 Yamashita, T. et al. Activation of lipogenic pathway correlates with cell proliferation and poor prognosis in hepatocellular carcinoma. Journal of hepatology 50, 100-110, doi:10.1016/j.jhep.2008.07.036 (2009).

41 Calvisi, D. F. et al. Increased lipogenesis, induced by AKT-mTORC1-RPS6 signaling, promotes development of human hepatocellular carcinoma. Gastroenterology 140, 1071-1083, doi:10.1053/j.gastro.2010.12.006 (2011). 
794

795

796

797

798

799

800

801

802

803

804

805

806

807

808

809

810

811

812

813

814

815

816

817

818

819

820

821

822

823

824

825

826

827

828

829

830

831

832

833

834

835

836

837

838

839

840

841

842

843

42 Svensson, R. U. et al. Inhibition of acetyl-CoA carboxylase suppresses fatty acid synthesis and tumor growth of non-small-cell lung cancer in preclinical models. Nature medicine 22, 1108-1119, doi:10.1038/nm.4181 (2016).

43 Lally, J. S. V. et al. Inhibition of Acetyl-CoA Carboxylase by Phosphorylation or the Inhibitor ND654 Suppresses Lipogenesis and Hepatocellular Carcinoma. Cell Metab 29, 174-182.e175, doi:10.1016/j.cmet.2018.08.020 (2019).

44 Camarda, R. et al. Inhibition of fatty acid oxidation as a therapy for MYC-overexpressing triplenegative breast cancer. Nature medicine 22, 427-432, doi:10.1038/nm.4055 (2016).

45 Padanad, M. S. et al. Fatty Acid Oxidation Mediated by Acyl-CoA Synthetase Long Chain 3 Is Required for Mutant KRAS Lung Tumorigenesis. Cell reports 16, 1614-1628, doi:10.1016/j.celrep.2016.07.009 (2016).

46 Duman, C. et al. Acyl-CoA-Binding Protein Drives Glioblastoma Tumorigenesis by Sustaining Fatty Acid Oxidation. Cell Metab 30, 274-289.e275, doi:10.1016/j.cmet.2019.04.004 (2019).

47 Schafer, Z. T. et al. Antioxidant and oncogene rescue of metabolic defects caused by loss of matrix attachment. Nature 461, 109-113, doi:10.1038/nature08268 (2009).

48 Zaugg, K. et al. Carnitine palmitoyltransferase 1C promotes cell survival and tumor growth under conditions of metabolic stress. Genes \& development 25, 1041-1051, doi:10.1101/gad.1987211 (2011).

49 Carracedo, A., Cantley, L. C. \& Pandolfi, P. P. Cancer metabolism: fatty acid oxidation in the limelight. Nat Rev Cancer 13, 227-232, doi:10.1038/nrc3483 (2013).

50 Gomes, L. C., Di Benedetto, G. \& Scorrano, L. During autophagy mitochondria elongate, are spared from degradation and sustain cell viability. Nat Cell Biol 13, 589-598, doi:10.1038/ncb2220 (2011).

51 Rambold, A. S., Kostelecky, B., Elia, N. \& Lippincott-Schwartz, J. Tubular network formation protects mitochondrial from autophagosomal degradation during nutrient starvation. Proc Natl Acad Sci U S A 108, 10190-10195 (2011).

52 Lee, Y. K., Lee, H. Y., Hanna, R. A. \& Gustafsson, A. B. Mitochondrial autophagy by Bnip3 involves Drp1-mediated mitochondrial fission and recruitment of Parkin in cardiac myocytes. Am. j. Physiol. Heart Circ. Physiol. 301, H1924-1931 (2011).

53 Rambold, A. S., Cohen, S. \& Lippincott-Schwartz, J. Fatty acid trafficking in starved cells: regulation by lipid droplet lipolysis, autophagy, and mitochondrial fusion dynamics. Dev. Cell 32, 678-692 (2015).

54 Rossignol, R. et al. Energy substrate modulates mitochondrial structure and oxidative capacity in cancer cells. Cancer Res. 64, 985-993 (2004).

55 Svensson, R. U. \& Shaw, R. J. Lipid Synthesis Is a Metabolic Liability of Non-Small Cell Lung Cancer. Cold Spring Harbor symposia on quantitative biology 81, 93-103, doi:10.1101/sqb.2016.81.030874 (2016).

56 Jiang, $\mathrm{H}$. et al. Genetic variants in de novo lipogenic pathway genes predict the prognosis of surgically-treated hepatocellular carcinoma. Scientific reports 5, 9536, doi:10.1038/srep09536 (2015).

57 Ferraro, G. B. et al. FATTY ACID SYNTHESIS IS REQUIRED FOR BREAST CANCER BRAIN METASTASIS. Nat Cancer 2, 414-428, doi:10.1038/s43018-021-00183-y (2021).

58 Ventura, R. et al. Inhibition of de novo Palmitate Synthesis by Fatty Acid Synthase Induces Apoptosis in Tumor Cells by Remodeling Cell Membranes, Inhibiting Signaling Pathways, and Reprogramming Gene Expression. EBioMedicine 2, 808-824, doi:10.1016/j.ebiom.2015.06.020 (2015).

59 Heuer, T. S. et al. FASN Inhibition and Taxane Treatment Combine to Enhance Anti-tumor Efficacy in Diverse Xenograft Tumor Models through Disruption of Tubulin Palmitoylation and Microtubule Organization and FASN Inhibition-Mediated Effects on Oncogenic Signaling and Gene Expression. EBioMedicine 16, 51-62, doi:10.1016/j.ebiom.2016.12.012 (2017). 
84460 Montesdeoca, N., López, M., Ariza, X., Herrero, L. \& Makowski, K. Inhibitors of lipogenic enzymes as a potential therapy against cancer. FASEB journal : official publication of the Federation of (2020).

61 Benador, I. Y. et al. Mitochondria Bound to Lipid Droplets Have Unique Bioenergetics, Composition, and Dynamics that Support Lipid Droplet Expansion. Cell Metab 27, 869-885.e866, doi:10.1016/j.cmet.2018.03.003 (2018).

62 Mizushima, N. \& Levine, B. Autophagy in mammalian development and differentiation. Nat. Cell Biol. 12, 823-830 (2010).

63 Torre, D., Lachmann, A. \& Ma'ayan, A. BioJupies: Automated Generation of Interactive Notebooks for RNA-Seq Data Analysis in the Cloud. Cell Syst 7, 556-561.e553, doi:10.1016/j.cels.2018.10.007 (2018).

64 Cerami, E. et al. The cBio cancer genomics portal: an open platform for exploring multidimensional cancer genomics data. Cancer Discov 2, 401-404, doi:10.1158/2159-8290.Cd12-0095 (2012).

65 Gao, J. et al. Integrative analysis of complex cancer genomics and clinical profiles using the cBioPortal. Sci Signal 6, pl1, doi:10.1126/scisignal.2004088 (2013). 


\section{Supplementary Files}

This is a list of supplementary files associated with this preprint. Click to download.

- Supplementallnformation.pdf

- nrreportingsummaryNCOMMS2138698.pdf 